\title{
Review of stochastic differential equations in statistical arbitrage pairs trading
}

\section{Introduction}

Since the seminal studies of Thiele (1880), Bachelier (1900), Einstein (1905) and von Smoluchowski (1906), the use of stochastic differential equations in science, engineering and economics has expanded rapidly (Bodo et al. 1987, Sharp 1990). More recently, in statistical arbitrage pairs trading, interest in advanced time-series modeling with stochastic differential equations has grown strongly, mainly due to increased activity on financial markets, the steady growth of computing power, and immense amounts of data at higher frequencies. The statistical arbitrage pairs trading strategy was introduced by Gatev et al. (1999) and Gatev et al. (2006) and consists of two time periods - formation and trading. In the formation period, pairs of strongly related stocks are formed by methods of time-series analysis. In the trading period, these pairs are monitored to detect any potential divergence in their price movements. If sufficiently large divergence occurs, the undervalued asset is bought and the overvalued asset is sold short, betting on a subsequent convergence of the two assets.

By construction, the pairs trading strategy typically relies on the mean-reverting tendency of spreads ${ }^{1}$ and its reliable quantification. For this purpose, stochastic differential equations are utilized to model spread characteristics and explain their potential mean-reverting behavior. Specifically, stochastic spread modeling focuses on the "two main issues in implementing a pairs trading strategy" (Focardi et al. 2016): In the formation period, pairs are selected based on estimated model parameters, e.g., strength of mean-reversion, and as such, matching of pairs is

* University of Erlangen-Nürnberg, Department of Statistics and Econometrics, Lange Gasse 20, 90403 Nürnberg, Germany, e-mail: sylvia.endres@fau.de.

${ }^{1}$ Methods to measure the spread include the difference of (log-)prices and the difference of (cumulated) (log-)returns of two stocks. Only few studies use other measures of relative mispricing, e.g., the price ratio of two assets. 
clearly improved, rendering model-free metrics like the Euclidean distance unnecessary. In the trading period, investment decisions are determined based on predictions obtained from the stochastic spread model, replacing traditional rules of thumb by optimized signals.

The majority of studies focuses on the trading period - in other words, it is assumed that pairs have already been selected and the goal is to derive optimal trading decisions. Hereby, two approaches are distinguished: In the first approach, optimized trading signals are derived using analytic frameworks with closed-form solutions. The second approach applies dynamic programming techniques to solve portfolio optimization problems (see Krauss 2017). The present survey follows this structure and hence organizes the works along two dimensions, i.e., (i) the stochastic model used to explain the spread and (ii) the trading optimization approach (analytic vs. dynamic programming) based on the stochastic spread model.

In total, the literature on statistical arbitrage pairs trading with stochastic differential equations consists of more than 80 studies. Across the wide range of stochastic models and their underlying mathematics as well as the diversity of statistical arbitrage frameworks, there remains lack of a holistic look at the research field from all the different angles. As of today, no academic work has yet consolidated and organized the extensive available knowledge.

In the present paper, we fill this void by surveying the multitude of available references. Hereby, our contribution to research is threefold. First, we provide a comprehensive literature review that systematically categorizes the large body of work into five main strands of stochastic spread models: the Ornstein-Uhlenbeck model, extended Ornstein-Uhlenbeck models, advanced mean-reverting diffusion models, diffusion models with a non-stationary component, and other models. Table 1 provides an overview of these models, their corresponding stochastic differential equations, and representative studies per category.

For each model, the approaches to derive optimized trading decisions are discussed thoroughly in consistent terminology and notation in sections 2-6. The corresponding tables $2-6$ summarize the main aspects per section. Second, we discuss the major papers in detail and assess the relative strengths and weaknesses according to model and approach. Hereby, we provide valuable insights into the current state of research with its key fields and main limitations. Third, we reveal directions for further research and promising future studies within each category.

The remainder of this study is structured as follows. Section 2 covers the Ornstein-Uhlenbeck model, section 3 extended Ornstein-Uhlenbeck models, section 4 advanced mean- reverting diffusion models. Diffusion models with a non-stationary component are reviewed in section 5 and other models in section 6 . Section 7 concludes and summarizes the main results. 


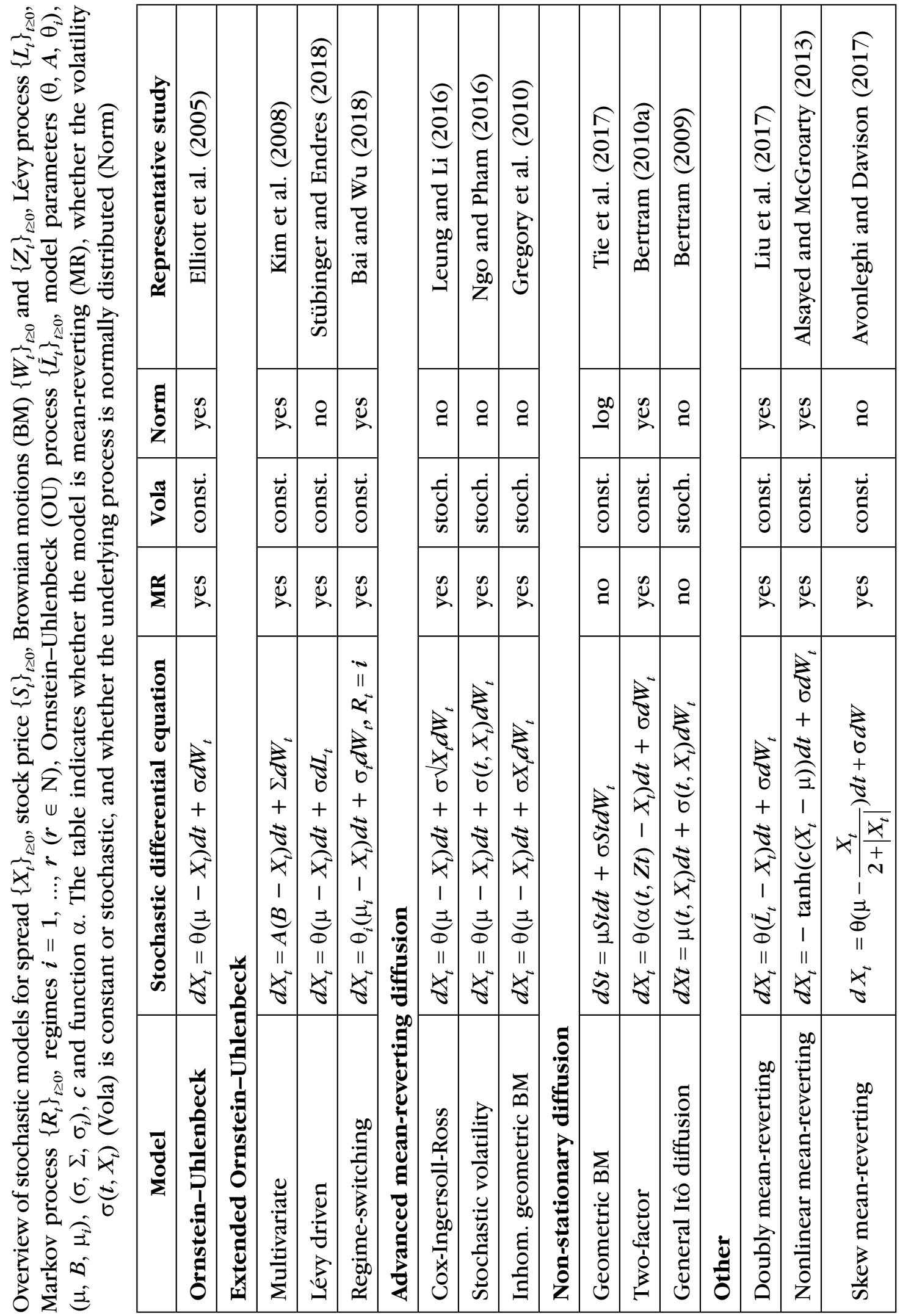




\section{Ornstein-Uhlenbeck model}

The Ornstein-Uhlenbeck (OU) model in pairs trading was proposed by Elliott et al. (2005). The authors laid the foundation ${ }^{2}$ for prediction and decision making based on this model (Zeng and Lee 2014). Nowadays, the majority of pairs trading studies use the OU model to explain the spread dynamics. For stock prices $S_{1}(t)$ and $S_{2}(t)$, the spread $X_{t}$ is typically calculated by $X_{t}=S_{1}(t)-S_{2}(t)$ or $X_{t}=\ln S_{1}(t)-\ln S_{2}(t)^{3}$ and follows

$$
d X_{t}=\theta\left(\mu-X_{t}\right) d t+\sigma d W_{t}
$$

with mean-reversion level (i.e., long-term mean, equilibrium level) $\mu$, meanreversion rate or speed $\theta$, volatility $\sigma$, and Brownian motion $\left\{W_{t}\right\}_{t \geq 0}$. The meanreversion level $\mu$ is a key element of the pairs trading strategy - the spread reverts to this level at rate $\theta$ and strategies usually take advantage of this behavior. The half-life $\boldsymbol{b}$ measures the time taken by the process to move halfway back to its equilibrium after divergence and is calculated by

$$
b=\frac{\ln 2}{\theta}
$$

The conditional distribution of the OU process is

$$
\left(X_{t} \mid X_{0}=x\right) \sim \mathcal{N}\left(\mu+(x-\mu) e^{-\theta t}, \frac{\sigma^{2}}{2 \theta}\left(1-e^{-2 \theta t}\right)\right)
$$

and in the limit

$$
X \sim N\left(\mu, \frac{\sigma^{2}}{2 \theta}\right)
$$

Transforming the OU process (1) into a dimensionless system via $Z_{t}=\left(X_{t}-\mu\right) \sqrt{2} \theta / \sigma$ (see, e.g., Zeng and Lee 2014) yields the dimensionless OU process

$$
d Z_{t}=-Z_{t} d t+\sqrt{2} d W_{t}
$$

${ }^{2}$ As of 2018-09-16, there are more than 285 citations on Google Scholar for Elliott et al. (2005).

${ }^{3}$ According to Do et al. (2006), the spread should be defined by $X_{t}=\ln \left(S_{1}(t)\right) \ln \left(S_{2}(t)\right)$ to remove the implicitly assumed restriction of "return parity". Assume that, in one unit of time, stocks 1 and 2 both return $r(r \in \mathbb{R})$. Then, $\ln \left(S_{1}(t+1)\right)-\ln \left(S_{2}(t+1)\right)=\left(\ln \left(S_{1}(t)\right)+r\right)-\left(\ln \left(S_{2}(t)\right)+r\right)=$ $=\ln \left(S_{1}(t)\right)-\ln \left(S_{2}(t)\right)$. 
with long-term mean zero. The first-passage time density in the dimensionless system is explicitly known (see, e.g., Göncü and Akyildirim 2016a)

$$
f_{0, c}(t)=\sqrt{\frac{2}{\pi}} \frac{|c| e^{-t}}{\left(1-e^{-2 t}\right)^{3 / 2}} \exp \left(-\frac{c^{2} e^{-2 t}}{2\left(1-e^{-2 t}\right)}\right), t>0
$$

Hereby, $f_{0, c}$ is the density of the first-passage time

$$
\tau_{0, c}=\inf \left\{t \geq 0, Z_{t}=0 \mid Z_{0}=c\right\}
$$

which is the time until $Z_{t}$ reaches its mean-reversion level 0 when starting in $c$. In the original OU process (1), the level $c$ corresponds to $\mu+c(\sigma / \sqrt{2} \theta)$ (see Göncü and Akyildirim 2016a).

Pairs trading decisions from the calibrated OU model can be optimized in the formation and trading period. In the formation period, the OU model parameters can be used for pairs selection. Favorable pairs are, e.g., (i) pairs with high meanreversion speed $\theta$ or low half-life $b$ (see equation 2) because they revert back to their equilibrium level fast ${ }^{4}$, (ii) pairs with high volatility $\sigma$ or high equilibrium standard deviation $\sigma_{e q}$ (see equation 3 ) because they create many trading opportunities. In the trading period, entry and exit decisions are optimized based on the calibrated OU model. Hereby, the basis is a rule of thumb ('two- sigma rule') explained by Gatev et al. (2006) - pairs are opened when the spread deviates by more than two historical standard deviations $s_{t}$ from its moving average $\mu_{t}$, i.e., the spread crosses $\mu_{t} \pm 2 s_{t}$, and closed when it reverts back to $\mu_{t}$. The studies surveyed in this subsection modify or replace this rule of thumb by advanced signals obtained from the OU model. We discuss the three approaches of literature to optimize trading decisions: analytic (subsection 2.1), dynamic programming (subsection 2.2), and other (subsection 2.3). Table 2 provides an overview of the relevant works which are discussed in the following part.

Table 2

Ornstein-Uhlenbeck model

\begin{tabular}{|l|l|}
\hline \multicolumn{1}{|c|}{ Approach } & \multicolumn{1}{c|}{ Study } \\
\hline \multicolumn{2}{|c|}{ 2.1. Analytic approach } \\
\hline 2.1.1. Baseline approach & Elliott et al. (2005) \\
\hline $\begin{array}{l}\text { Generalizations of the baseline } \\
\text { approach }\end{array}$ & $\begin{array}{l}\text { Do et al. (2006), Triantafyllopoulos and Montana } \\
(2011)\end{array}$ \\
\hline Further analytic results & Rampertshammer (2007) \\
\hline
\end{tabular}

\footnotetext{
${ }^{4}$ High mean-reversion speed or low half-life are equivalent metrics for pairs selection.
} 
Table 2 cont.

\begin{tabular}{|c|c|}
\hline Approach & Study \\
\hline $\begin{array}{l}\text { Applications of the baseline } \\
\text { approach }\end{array}$ & $\begin{array}{l}\text { Baronyan et al. (2010), Dunis et al. (2010), } \\
\text { Bogomolov (2011), Kim (2011), Nobrega and } \\
\text { Oliveira (2013), Diamond (2014), Fanelli and } \\
\text { Lesca (2014), Nobrega and Oliveira (2014), Kang } \\
\text { and Leung (2017), Yang et al. (2017), Blázquez } \\
\text { and Román (2018), Psaradellis et al. (2018) }\end{array}$ \\
\hline Further approaches & $\begin{array}{l}\text { Kanamura et al. (2010), Bogomolov (2013), } \\
\text { Temnov (2015) }\end{array}$ \\
\hline 2.1.2. Optimal trading thresholds & Bertram (2010b) \\
\hline $\begin{array}{l}\text { Empirical applications of Bertram } \\
\text { (2010b) }\end{array}$ & $\begin{array}{l}\text { Cummins (2010), Bucca and Cummins (2011), } \\
\text { Cummins and Bucca (2012) }\end{array}$ \\
\hline $\begin{array}{l}\text { Further enhancements of Bertram } \\
\text { (2010b) }\end{array}$ & $\begin{array}{l}\text { Zeng and Lee (2014), Göncü and Akyildirim } \\
\text { (2016b), Baviera and Baldi (2018) }\end{array}$ \\
\hline \multicolumn{2}{|c|}{ 2.2. Dynamic programming } \\
\hline $\begin{array}{l}\text { 2.2.1. Optimal investment } \\
\text { allocation }\end{array}$ & $\begin{array}{l}\text { Boguslavsky and Boguslavskaya (2004), Jurek and } \\
\text { Yang (2007), Mudchanatongsuk et al. (2008) }\end{array}$ \\
\hline Extension for time-dependency & Charalambous et al. (2015) \\
\hline Continuous-time cointegration & $\begin{array}{l}\text { Liu and Timmermann (2013), Tourin and Yan } \\
\text { (2013) }\end{array}$ \\
\hline $\begin{array}{l}\text { Extending continuous-time } \\
\text { cointegration }\end{array}$ & $\begin{array}{l}\text { Figuerola-Ferretti et al. (2015), Angoshtari } \\
(2016), \text { Li and Tourin (2016), Figuerola-Ferretti } \\
\text { et al. (2017) }\end{array}$ \\
\hline 2.2.2. Optimal timing of trades & Zhang and Zhang (2008) \\
\hline Incorporating stop-loss limits & $\begin{array}{l}\text { Ekström et al. (2011), Song and Zhang (2013), } \\
\text { Lindberg (2014), Kuo et al. (2015), Leung and Li } \\
\text { (2015), Li (2015), Leung and Li (2016) }\end{array}$ \\
\hline $\begin{array}{l}\text { Further enhancements on optimal } \\
\text { timing: Finite horizon, multiple } \\
\text { regimes, cointegration, model } \\
\text { uncertainty }\end{array}$ & $\begin{array}{l}\text { Dourban and Yedidsion (2015), Lei and Xu } \\
\text { (2015), Suzuki (2016), Kitapbayev and Leung } \\
\text { (2017), Yoshikawa (2017), Kitapbayev and Leung } \\
\text { (2018), Suzuki (2018) }\end{array}$ \\
\hline \multicolumn{2}{|c|}{ 2.3. Other approaches } \\
\hline Principal component analysis & $\begin{array}{l}\text { Avellaneda and Lee (2010), Yeo and Papanicolaou } \\
\text { (2017), Burks et al. (2018) }\end{array}$ \\
\hline Relativistic statistical arbitrage & Wissner-Gross and Freer (2010) \\
\hline Penalized likelihood approach & Zhang et al. (2018) \\
\hline
\end{tabular}




\subsection{Analytic approach}

The key idea of the analytic approach is to obtain optimized trading thresholds using closed form solutions.

\subsubsection{Baseline approach}

Elliott et al. (2005) were the first authors to apply the OU model to explain the spread $X_{t}=S_{1}(t)-S_{2}(t)$. A pairs trade is opened when the dimensionless OU process $Z_{t}$ (see equation 4 ) crosses a threshold $c>0$, i.e., reaches an extreme value. To determine the optimal exit timing, the authors suggest to use the first-passage time $\tau_{0, c}$, since the corresponding probability density function $f_{0, c}$ is explicitly known. The trade is exited at a fixed time $\tilde{t}$, where $f_{0, c}(t)$ has a maximum value and thus $Z_{t}$ reaches its long-term mean 0 with greatest probability. For the original OU process (see equation 1 ), this means that a trade is entered when $X_{t}$ crosses $\mu \pm c(\sigma / \sqrt{2} \theta)$ and exited $T=\frac{1}{\theta} \tilde{t}$ times later - at the most likely time at which $X_{t}$ reaches its mean-reversion level $\mu$. However, the threshold $c$ is not further specified by the authors. Further, Cummins and Bucca (2012) point out that the lengths of the trade cycles are uncertain and not directly considered in the strategy.

Do et al. (2006) generalize the approach of Elliott et al. (2005) and model the spread $X_{t}$ as the difference of returns instead of prices. They additionally include a loading matrix $\Gamma$ and an exogenous vector $U_{t}$ for modeling the equilibrium. The modeling framework is similar to Elliott et al. (2005) and the dynamics of $X_{t}$ are governed by an OU process. A pairs trade is opened when the cumulated spread crosses a certain threshold and closed when it reaches the long run level of the spread. However, no explicit threshold for market entry is given. Triantafyllopoulos and Montana (2011) extend the state-space framework of Elliott et al. (2005) for time-dependent model parameters, thus gaining flexibility and being able to quickly adapt to changes in the data. Further analytic trading frameworks based on the OU model are discussed by Rampertshammer (2007).

In subsequent years, various studies optimize the strategy with the calibrated OU model based on the framework of Elliott et al. (2005). In the formation period, Dunis et al. (2010), Kim (2011) and Blázquez and Román (2018) select pairs based on estimated OU model parameters, e.g., mean-reversion speed $\theta$, instead of simple distance metrics. In the trading period, the traditional 'two-sigma rule' by Gatev et al. (2006) is optimized by using the OU model parameters $\mu$ and $\sigma$ instead of moving average $\mu_{t}$ and historical standard deviation $s_{t}$. Baronyan et al. (2010), Bogomolov (2011), Diamond (2014), and Fanelli and Lesca (2014) open positions when the spread reaches an exteme value $\mu \pm k_{1} \sigma$ and exit them when the spread reverts back to $\mu \pm k_{2} \sigma$, choosing various values for $k_{1}$ and $k_{2}$. Other 
trading rules based on OU model parameters are constructed in Nobrega and Oliveira (2013), Nobrega and Oliveira (2014), Kang and Leung (2017), Yang et al. (2017), and Psaradellis et al. (2018). Apparently, there exist various different approaches for trading, which raise the question whether there is an optimal and completely model-driven rule.

Further analytic approaches are provided by Kanamura et al. (2010), Bogomolov (2013), and Temnov (2015). Kanamura et al. (2010) introduce a profit model for spread trading, taking advantage of the explicit first-passage time density of the OU process. Bogomolov (2013) proposes a nonparametric pairs trading approach and demonstrates its theoretical profitability for the OU process. Temnov (2015) develops a trading strategy based on an explicit formula for the running maximum of an OU process stopped at its maximum drawdown.

\subsubsection{Focusing on the role of time - optimal trading thresholds}

Analytic formulas for optimal trading are finally provided by Bertram (2010b). The spread is modeled by the OU process (1) with mean-reversion level $\mu=0^{5}$. A trade is entered at $X_{t}=a$ and exited at $X_{t}=m$ for $a<m$. A trade cycle is completed when the spread has reverted back to the starting value $a$. The time the spread needs to undergo this cycle is the total trade length $\tau=\tau_{m, a}+\tau_{a, m}$ with mean $E[\tau]$, variance $\operatorname{Var}[\tau]$, and $\tau_{m, a}=\inf \left\{t \geq 0 \mid X_{t} \geq m, X_{0}=a\right\}$. An optimal strategy is derived by maximizing two different objective functions - the expected return per unit of time

$$
\mu(a, m, c)=\frac{r(a, m, c)}{E[\tau]}
$$

and the expected Sharpe ratio per unit of time:

$$
S\left(a, m, c, r_{f}\right)=\frac{\mu(a, m, c)-\frac{r_{f}}{E[\tau]}}{E[\tau]}
$$

for return $r(a, m, c)=m-a-c$, transaction costs $c$, risk-free rate $r_{f}$, and return variance

$$
\sigma^{2}(a, m, c)=r(a, m, c)^{2} \operatorname{Var}[1 / \tau]=r(a, m, c)^{2} \operatorname{Var}[\tau] / E[\tau]^{3}
$$

The distribution of the first-passage time is known (see equation 5) and thus $E[\tau]$ and $\operatorname{Var}[\tau]$ can be calculated explicitly.

${ }^{5}$ Following Cummins and Bucca (2012), the zero-mean assumption is no issue in practice since the analytic results can be translated easily to a non-zero mean. 
As such, analytic formulas for $\mu(a, m, c)$ and $S\left(a, m, c, r_{f}\right)$ can be derived

$$
\mu(a, m, c)=\frac{\theta(m-a-c)}{\pi\left(\operatorname{Erfi}\left(\frac{m \sqrt{\theta}}{\sigma}\right)-\operatorname{Erfi}\left(\frac{a \sqrt{\theta}}{\sigma}\right)\right)}
$$

and $S\left(a, m, c, r_{f}\right)$ similarly. $\operatorname{Erfi}(x)$ denotes the imaginary error function. The optimal analytic trading thresholds $a$ and $m$ are received by maximizing these functions via differentiation. For the case of maximizing the expected return per unit of time, the following entry bound $a$ is obtained

$$
\begin{gathered}
a=-\frac{c}{4}-\frac{c^{2} \theta}{4\left(c^{3} \theta^{3}+24 c \theta^{2} \sigma^{2}-4 \sqrt{3 c^{4} \theta^{5} \sigma^{2}+36 c^{2} \theta^{4} \sigma^{4}}\right)^{1 / 3}}- \\
\frac{\left(c^{3} \theta^{3}+24 c \theta^{2} \sigma^{2}-4 \sqrt{3 c^{4} \theta^{5} \sigma^{2}+36 c^{2} \theta^{4} \sigma^{4}}\right)^{1 / 3}}{4 \theta}
\end{gathered}
$$

The optimal trading bounds are found to be symmetric around the meanreversion level of the process, i.e., $m=-a$. The approach by Bertram (2010b) has three advantages: First, the trading thresholds are optimal and model-driven classic rules of thumb are rendered unnecessary. Second, closed-form solutions allow for analytic investigations and computationally efficient frameworks even in the high-frequency context (see Krauss 2017). Third, the approach allows for consistent cross-comparison, i.e., different strategies can be compared since their deterministic returns are all normalized by the expected trade cycle time (see Cummins and Bucca 2012). For future research, Bertram (2010b) suggests to apply the method to non-Gaussian processes, e.g., processes driven by Lévy noise, although it is not clear whether comparable analytic results exist. Bucca and Cummins (2011) suggest to consider regime shifts in the long-run mean level of the spread series. The surveyed studies in subsections 3.2 and 3.3 focus on the latter two issues. Zeng and Lee (2014) criticize that fixed trading thresholds are not applicable in the long-run and propose to investigate time-dependent thresholds.

Empirical applications of the approach by Bertram (2010b) are found in Cummins (2010), Bucca and Cummins (2011), and Cummins and Bucca (2012). Cummins (2010) performs a comprehensive analysis on Irish stock exchange data. Bucca and Cummins (2011) conduct a model specification analysis on Brent and TD3 data. Cummins and Bucca (2012) provide a large application on oil based markets and control for data snooping bias.

Further enhancements of the approach by Bertram (2010b) are provided by Zeng and Lee (2014), Göncü and Akyildirim (2016b), and Baviera and Baldi (2018). 
Zeng and Lee (2014) derive optimal analytic trading thresholds for an OU process, considering first-passage times over a two-sided symmetric boundary. Göncü and Akyildirim (2016b) derive optimal trading bounds for another objective function than Bertram (2010b) - the probability of successful termination of the strategy. Baviera and Baldi (2018) extend the optimal strategy for two key elements of high-frequency trading - stop-loss and leverage.

\subsection{Dynamic programming}

Based on the OU model to explain the spread dynamics, another stream of literature focuses on dynamic programming techniques. The key idea of the dynamic programming approach is to solve an arbitrageur's dynamic portfolio optimization problem based on stochastic control theory. An investor with given preference specification ${ }^{6}$ can either trade a risky arbitrage opportunity $X_{t}$ or allocate capital to a risk-free asset $M_{t}$. The arbitrage opportunity, i.e., the mean-reverting spread $X_{t}$, is modeled by a stochastic differential equation.

\subsubsection{Optimal investment allocation}

A different angle to look at pairs trading is the problem of determining optimal portfolio holdings over time. The most cited paper $^{7}$ in this domain is Mudchanatongsuk et al. (2008). The authors assume that an investor either allocates capital to a risk-free asset $M_{t}$ with rate $r$ or to a stock pair with prices $S_{1}(t)$ and $S_{2}(t)$ and spread $X_{t}$, governed by the OU process. The portfolio weights of stocks 1 and 2 are denoted by $b_{1}(t)$ and $b_{2}(t)$ and it is required that $b_{1}(t)=-b_{2}(t)$, i.e., the pairs trading portfolio is dollar-neutral. A negative weight represents a short position in the respective stock. The portfolio value $V_{t}$ follows

$$
d V_{t}=V_{t}\left(b_{1}(t) \frac{d S_{1}(t)}{S_{1}(t)}+b_{2}(t) \frac{d S_{2}(t)}{S_{2}(t)}+\frac{d M_{t}}{M_{t}}\right)
$$

The objective is to maximize the expected utility from this portfolio at the final time $T$. For an investor with power utility, the optimization problem has the following form:

$$
\sup _{b_{1}(t)} E\left[\frac{1}{\gamma} V_{T}^{\gamma}\right]
$$

\footnotetext{
${ }^{6}$ Different utility functions for an investor with risk aversion $\gamma$ are considered: Power utility $u(x)=\frac{1}{\gamma} x^{\gamma}$, exponential utility $u(x)=e^{-\gamma x}$, logarithmic utility $u(x)=\ln (x)$, constant relative risk aversion (CRRA) utility, constant absolute risk aversion (CARA) utility, and Epstein-Zin utility.

${ }^{7}$ As of 2018-09-16, there are 85 citations on Google Scholar for Mudchanatongsuk et al. (2008).
} 
subject to: $V(0)=v_{0}, X(0)=x_{0}$

$$
\begin{gathered}
d X_{t}=\theta\left(\mu-X_{t}\right) d t+\sigma d W_{t} \\
d V_{t}=V_{t}\left(\left(h_{1}(t)\left(\theta\left(\mu-X_{t}\right)+\frac{1}{2} \sigma^{2}+\rho \eta \sigma\right)+r\right) d t+\sigma d W_{t}\right)
\end{gathered}
$$

with volatility $\eta$ of the dynamics of stock 2 and correlation $\rho$. The stochastic control problem (10) is solved using dynamic programming techniques - the optimal weight $\boldsymbol{b}_{1}^{*}(t)$ is obtained via the Hamilton-Jacobi-Bellman (HJB) equations as

$$
b_{1}^{*}(t, x)=\frac{1}{1-\gamma}\left(\beta(t)+2 x \alpha(t)-\frac{\theta(x-\mu)}{\sigma^{2}}+\frac{\rho \eta}{\sigma}+\frac{1}{2}\right)
$$

with functions $\alpha(t)$ and $\beta(t)$. The work of Mudchanatongsuk et al. (2008) lays the foundation for pairs trading based on stochastic control (see Lintilhac and Tourin 2017). The major contribution are optimal closed-form solutions for the portfolio holdings. However, there are two downsides associated with this approach, which particularly impact practical applications. First, transaction costs are not considered in the study. Second, the optimal strategy requires infinitesimal rebalance (see Suzuki 2016), i.e., positions have to be adjusted constantly according to equation (11). Jurek and Yang (2007) consider a similar setting as Mudchanatongsuk et al. (2008) and focus on an investor with constant relative risk aversion (CRRA) and Epstein-Zin utility. For CRRA utility and $\gamma=1$, they derive the optimal portfolio allocation by

$$
b^{*}\left(V_{t}, x\right)=\left(-\frac{\theta(x-\mu)}{\sigma^{2}}-\frac{r x}{\sigma^{2}}\right) V_{t}
$$

Boguslavsky and Boguslavskaya (2004) solve the optimal investment problem for an arbitrageur with power utility and a single risky asset that follows an OU process, i.e., the risk-free rate $r$ is assumed to be zero. Charalambous et al. (2015) provide an extension of Mudchanatongsuk et al. (2008) for time-dependent model parameters.

A common characteristic of the aforementioned studies is that they assume dollar-neutrality, i.e., the investor goes long one stock and short the other in equal dollar amounts. In recent years, some studies have dropped the dollar-neutrality assumption of classic pairs trading. By modeling two cointegrated assets in a continuous-time setting, the amounts invested in each position of the pairs trade are determined separately. The most cited $\operatorname{paper}^{8}$ in this domain is Tourin and Yan (2013).

\footnotetext{
${ }^{8}$ As of 2018-09-16, there are more than 40 citations on Google Scholar for Tourin and Yan (2013).
} 
The authors model the prices $S_{1}(t)$ and $S_{2}(t)$ of two cointegrated stocks for $t \in[0, T]$ by

$$
\begin{gathered}
d \ln S_{1}(t)=\left(\mu_{1}-\frac{\sigma_{1}^{2}}{2}+\delta z_{t}\right) d_{t}+\sigma_{1} d W_{1}(t) \\
d \ln S_{2}(t)=\left(\mu_{2}-\frac{\sigma_{2}^{2}}{2}\right) d_{t}+\sigma_{2} d W_{2}(t)
\end{gathered}
$$

with co-integrating vector $z_{t}$ :

$$
z_{t}=\alpha+\ln S_{1}(t)+\beta \ln S_{2}(t)
$$

They show that $z_{t}$ follows the OU process

$$
\begin{aligned}
d z_{t} & =\left(\mu_{1}-\frac{\sigma_{1}^{2}}{2}+\delta z_{t}\right) d_{t}+\sigma_{1} d W_{1}(t)+\beta\left(\mu_{2}-\frac{\sigma_{2}^{2}}{2}\right) d_{t}+\beta \sigma_{2} d W_{2}(t) \\
& =\theta\left(\mu-z_{t}\right) d t+\sigma d W_{t}
\end{aligned}
$$

with $\theta=-\delta, \mu=-1 / \delta\left(\mu_{1}-\sigma_{1}^{2} / 2+\beta\left(\mu_{2}-\sigma_{2}^{2} / 2\right)\right)$, and $\sigma=\sqrt{\sigma_{1}^{2}+\beta^{2} \sigma_{1}^{2}}$. The investor's portfolio $V_{s}$ consists of stocks 1 and 2 with weights $b_{1}(s)$ and $b_{2}(s)$ for $s \in[t, T]$ and a risk-free asset, for which the interest rate is set to 0 . The optimal strategy is specified by the following stochastic control problem

$$
\sup _{\left(b_{1}, b_{2}\right)} E\left[u\left(V_{T}\right)\right]
$$

The authors use exponential utility $u$ with risk aversion coefficient $\gamma>0$ since their ansatz does not work for power utility. Via the HJB equations, the optimal solutions $\left(\boldsymbol{b}_{1}^{*}, \boldsymbol{b}_{2}^{*}\right)$ for problem (14) are derived as:

$$
\begin{aligned}
& b_{1}^{*} s_{1}=\frac{\mu_{1}+\delta z}{\gamma \sigma_{1}^{2}}-\delta \frac{\mu_{1}+\delta z}{\gamma \sigma_{1}^{2}}(T-t)+\frac{1}{4} \frac{\delta^{2}\left(\sigma_{1}^{2}+\beta \sigma_{2}^{2}\right)}{\gamma \sigma_{1}^{2}}(T-t)^{2} \\
& b_{2}^{*} s_{2}=\frac{\mu_{2}}{\gamma \sigma_{2}^{2}}-\beta \delta \frac{\mu_{1}+\delta z}{\gamma \sigma_{1}^{2}}(T-t)+\frac{1}{4} \beta \frac{\delta^{2}\left(\sigma_{1}^{2}+\beta \sigma_{2}^{2}\right)}{\gamma \sigma_{1}^{2}}(T-t)^{2}
\end{aligned}
$$

As such, the optimal holdings at any time $t$ depend on the stock prices and the wealth at that time. Subsequently, the authors present an extension that incorporates correlations between the two stocks and derive the optimal control pair. The simplicity of the aforementioned formulas allows for a straightforward implementation of the optimal strategy, while the limitations of this approach are pointed out by Zhengqin (2014). First, the analytic solutions only hold if the 
risk-free rate of return is zero. Second, the ansatz is not applicable for other types of utility functions. Third, in real world markets where transaction costs exist, it is not possible to adjust the stock positions constantly to take the optimal holdings. Further investigation of the optimal investment problem for cointegrated assets under power utility is found in Liu and Timmermann (2013).

Li and Tourin (2016) extend the model by Tourin and Yan (2013) for timevarying volatility, replacing the constant volatility coefficients in equation (13) by $\sigma_{1}(t, x)=\sigma_{1} e^{\theta_{1} x}$ and $\sigma_{2}(t, x)=\sigma_{2} e^{\theta_{2} x}$. Further, they use power utility instead of exponential utility. However for this framework, the authors are unable to derive a fully explicit solution for the stochastic control problem. Figuerola-Ferretti et al. (2015) and Figuerola-Ferretti et al. (2017) extend upon Liu and Timmermann (2013), maximizing the portfolio value without explicit specification of the investor's preferences in an utility function. Instead, they identify a replicating portfolio based on option valuation and connect it to the optimal strategy. Angoshtari (2016) rests upon the work of Liu and Timmermann (2013), deriving a theoretical justification for the practice of market-neutral pairs trading based on two cointegrated assets.

\subsubsection{Optimal timing of trades}

Besides the problem of optimal investment allocation, another stream of studies places focus on the strategy's optimal timing, determined with dynamic programming techniques. The baseline approach by Zhang and Zhang (2008) aims at maximizing a discounted reward function by sequentially buying and selling a mean-reverting asset $X_{t}=\ln S_{t}$ following the OU process. A position is bought at $\tau_{1}$, sold at $\sigma_{1}$, bought again at $\tau_{2}$, etc., leading to the following sequence of stopping times:

$$
0 \leq \tau_{1} \leq \sigma_{1} \leq \tau_{2} \leq \sigma_{2} \leq \ldots
$$

If the initial net positions is flat $(i=0)$, the decision sequence is $\Lambda_{0}=\left(\tau_{1}, \sigma_{1}, \tau_{2}, \sigma_{2}, \ldots\right)$, for an initial long position $(i=1)$, it is $\Lambda_{1}=\left(\sigma_{1}, \tau_{2}, \sigma_{2}, \tau_{3}, \ldots\right)$. For discount factor $\rho>0$ and slippage $0<K<1$, the authors maximize the discounted reward function

$$
V_{i}(x)=\sup _{\Lambda_{i}} J_{i}\left(x, \Lambda_{i}\right)
$$

for

$$
J_{0}\left(x, \Lambda_{0}\right)=E\left[\sum_{n=1}^{\infty}\left[e^{-\rho \sigma_{n}} S_{\sigma_{n}}(1-K)-e^{-\rho \tau_{n}} S_{\tau_{n}}(1+K)\right]\right]
$$


and $J_{1}\left(x, \Lambda_{1}\right)$ similarly. The authors show that a threshold pair $\left(x_{1}, x_{2}\right)$, obtained by solving two quasi-algebraic equations, leads to the optimal stopping times. The low threshold corresponds to the buy point, the high threshold to the sell point. Zhang and Zhang (2008) lay the foundation for optimal timing strategies based on stochastic control, allowing infinitesimal sequential buying and selling subject to slippage cost. However, buying and selling of positions at exactly the same time (see equation 15) is not possible in real world markets. Moreover, the authors only allow for two regimes, i.e., buy and sell, and the approach could be extended for a third regime, i.e., short the position (see Ngo and Pham 2016).

Integration of stop-loss thresholds into the optimal timing strategy can be found in various studies, i.e., Ekström et al. (2011), Song and Zhang (2013), Lindberg (2014), Kuo et al. (2015), Leung and Li (2015), Li (2015), and Leung and Li (2016).

In recent years, some studies present further enhancements to optimal timing strategies. Dourban and Yedidsion (2015), Kitapbayev and Leung (2017) and Kitapbayev and Leung (2018) study optimal stopping problems over finite horizons. However, they are not able to derive closed-form solutions. Suzuki (2016) and Suzuki (2018) focus on optimal switching over multiple regimes: no holding of stocks, long in the first stock and short in the second, and vice-versa. Lei and Xu (2015) study the optimal timing problem for two co-integrated assets whose cointegrating vector follows the OU process. Yoshikawa (2017) incorporate model uncertainty into the optimal boundary framework with the use of relative entropy.

\subsection{Other approaches}

This subsection summarizes other approaches based on the OU model with limited relation to the aforementioned references, covering principal component analysis, relativistic statistical arbitrage, and the penalized likelihood approach.

Principal component analysis. Avellaneda and Lee (2010) $\operatorname{model}$ the $\operatorname{spread} X_{t}$, defined by

$$
X_{t}=\ln \frac{S_{1}(t)}{S_{1}(0)}-\beta \ln \frac{S_{2}(t)}{S_{2}(0)}-\alpha t
$$

as an OU process. Based on this model, they generate trading signals for meanreverting portfolios with the aid of Principal Components Analysis (PCA) and exchange-traded funds. Motivated by this study, Yeo and Papanicolaou (2017) analyze the risk due to mis-estimation of mean-reversion based on Principal Components. Burks et al. (2018) use the framework of Avellaneda and Lee (2010) and analyze how systemic illiquidity affects mean-reverting trading strategies. 
Relativistic statistical arbitrage. Wissner-Gross and Freer (2010) describe the cointegrating linear combination between two correlated assets by the OU process and develop a relativistic generalization of statistical arbitrage trading strategies.

Penalized likelibood approach. Zhang et al. (2018) construct mean-reverting portfolios that follow an OU process via penalized likelihood estimation. Simultaneously, portfolios with desirable characteristics, i.e., high mean-reversion and low variance, are formed. The nonconvex optimization problem is solved by an algorithm based on partial minimization. Interestingly, the approach allows simultaneous portfolio selection and OU model calibration in one step. However, the study lacks specific trading rules.

Despite the obvious advantages of the OU model - simplicity, analytic tractability and the ability to explain the important mean-reverting property - it has several deficiencies and is not able to completely describe the reality of the spread process (see, e.g., Avonleghi and Davison 2017). The first downside is the constant volatility assumption. According to Pilipovic (2007) and Avonleghi and Davison (2017), eventful market news sometimes influence prices strongly and lead to increased volatility. The second downside is the Gaussian nature of the OU process. According to various studies (see, e.g., Bertram 2010b), financial data displays non-Gaussian behavior. The third downside is the constant equilibrium level of the OU process. In practice, a stochastic or at least time-dependent mean level is more realistic (see, e.g., Liu et al. 2017). Some of these disadvantages are compensated by extensions of the classic OU process, i.e., multivariate, Lévy driven, and regime-switching OU models.

\section{Extended Ornstein-Uhlenbeck models}

In recent years, extended Ornstein-Uhlenbeck models have been used to account for stylized facts of financial return series, e.g., correlations, fat tails, and regime-switches. The relevant works are summarized in Table 3.

\subsection{Multivariate Ornstein-Uhlenbeck model}

The assumption that there exists only one mean-reverting asset is very restrictive since a robust spread construction methodology could be used for various related spreads (see Jurek and Yang 2007). Combining multiple assets assures greater diversification (Jurek and Yang 2007, Kim et al. 2008) and allows to exploit co-moving patterns (Liu et al. 2017) and common interactions (Endres and Stübinger 2019b) between spreads. In this respect, multivariate OU models are used to construct portfolios of multiple correlated spreads. We consider this framework under the multivariate pairs trading umbrella. 
Table 3

Extended Ornstein-Uhlenbeck models

\begin{tabular}{|c|c|}
\hline Model & Study \\
\hline \multicolumn{2}{|c|}{ 3.1. Multivariate Ornstein-Uhlenbeck model } \\
\hline \multicolumn{2}{|c|}{ 3.1.1. Analytic approach } \\
\hline $\begin{array}{l}\text { Optimal trading thresholds: Decisions } \\
\text { based on first-passage times }\end{array}$ & Rampertshammer (2007) \\
\hline \multicolumn{2}{|c|}{ 3.1.2. Dynamic programming } \\
\hline $\begin{array}{l}\text { Optimal investment allocation: Optimal } \\
\text { portfolio holdings, continuous-time } \\
\text { cointegration, enhancements for time- } \\
\text { consistency and robustness }\end{array}$ & $\begin{array}{l}\text { Kim et al. (2008), Chiu and Wong (2011), } \\
\text { Chiu and Wong (2015), Lintilhac and } \\
\text { Tourin (2017), Yamamoto and Hibiki } \\
\text { (2017), Chiu and Wong (2018) }\end{array}$ \\
\hline \multicolumn{2}{|c|}{ 3.2. Lévy driven Ornstein-Uhlenbeck model } \\
\hline \multicolumn{2}{|c|}{ 3.2.1. Analytic approach } \\
\hline $\begin{array}{l}\text { Model-driven decisions: Optimizing for- } \\
\text { mation and trading period }\end{array}$ & Stübinger and Endres (2018) \\
\hline $\begin{array}{l}\text { Optimal trading thresholds: Decisions } \\
\text { based on first-passage time results }\end{array}$ & $\begin{array}{l}\text { Göncü and Akyildirim (2016a), Endres } \\
\text { and Stübinger (2019b) }\end{array}$ \\
\hline \multicolumn{2}{|c|}{ 3.2.2. Dynamic programming } \\
\hline $\begin{array}{l}\text { Optimal timing of trades: Optimal liquida- } \\
\text { tion, stop-loss thresholds }\end{array}$ & Larsson et al. (2013) \\
\hline \multicolumn{2}{|c|}{ 3.3. Regime-switching Ornstein-Uhlenbeck model } \\
\hline \multicolumn{2}{|c|}{ 3.3.1. Analytic approach } \\
\hline $\begin{array}{l}\text { Optimal trading thresholds: Maximum } \\
\text { expected return per unit of time }\end{array}$ & Bai and Wu (2018) \\
\hline \multicolumn{2}{|c|}{ 3.3.2. Dynamic programming } \\
\hline $\begin{array}{l}\text { Optimal investment allocation: Optimal } \\
\text { weights, logarithmic utility }\end{array}$ & Altay et al. (2017) \\
\hline \multicolumn{2}{|c|}{ 3.3.3. Other approaches } \\
\hline $\begin{array}{l}\text { Model-based trading rules: Two-state } \\
\text { model, different trading strategies }\end{array}$ & Yang et al. (2016) \\
\hline $\begin{array}{l}\text { Regime classification algorithm: Flexible } \\
\text { number of regimes }\end{array}$ & Endres and Stübinger (2019a) \\
\hline
\end{tabular}


The multivariate spread $X_{t}=\left(X_{1}, \ldots, X_{N}\right)$ consists of $N$ correlated spreads and follows

$$
d X_{t}=A\left(B-X_{t}\right) d t+\Sigma d W_{t}
$$

with $A \in \mathbb{R}^{N \times N}, B \in \mathbb{R}^{N}$, vector $\left\{W_{t}\right\}_{t \geq 0}$ of independent univariate standard Brownian motions, and positive definite covariance matrix

$$
\Sigma=\left(\begin{array}{ccc}
\sigma_{11} & \cdots & \sigma_{1 N} \\
\vdots & \ddots & \vdots \\
\sigma_{N 1} & \cdots & \sigma_{N N}
\end{array}\right)
$$

Table 3 provides an overview of the relevant works applying a multivariate OU model to construct portfolios of multiple correlated spreads. As in the previous section, we first discuss the analytic approach before dynamic programming techniques are presented.

\subsubsection{Optimal trading thresholds - analytic approach}

Rampertshammer (2007) provides analytic results on the OU model and corresponding first-passage times in a multivariate framework. The author models the spreads by equation (18) and chooses $B \equiv 0$, so the process reverts around $0^{9}$. Trades are opened when spreads diverge sufficiently large from their historical equilibrium, i.e., cross thresholds $a$ or $m, a<m$, and closed when their equilibrium is re-established, i.e., at time $\tau=\inf \left\{t \geq 0 \mid X_{t}=0\right\}$. To determine the optimal thresholds $a$ and $m$, a first-passage time problem needs to be solved. However, the author points out that it is not possible to solve the problem for the multivariate process analytically and suggests to use numerical methods. The only way would be to assume independence between the different spreads, but then the problem reduces to the modeling of separate univariate OU processes. Based on the results of Rampertshammer (2007), research should further investigate the problem of determining explicit optimal trading thresholds in the multivariate framework. Moreover, the extent of correlations among spreads in real world data should be analyzed to identify the practical relevance of multivariate modeling compared to the much simpler univariate approach.

\subsubsection{Optimal investment allocation - dynamic programming}

Kim et al. (2008) consider a dynamic trading strategy that allocates capital over a portfolio $V_{t}$ of multiple correlated spreads $X_{t}=\left(X_{1}, \ldots, X_{N}\right)$ and a risk-free asset.

\footnotetext{
9 The zero-mean assumption is no issue since the results could also be translated to a non-zero mean.
} 
The number of units of the spreads at time $t$ is denoted by $b(t)=\left(b_{1}(t), \ldots, b_{N}(t)\right)$. Assuming $X_{t}$ follows the multivariate OU model (18), the problem of determining optimal portfolio holdings over a finite horizon for CRRA and CARA utilities is investigated:

$$
\sup _{b} E\left[u\left(V_{T}\right) \mid X(t)=x_{t}, V(t)=v_{t}\right]
$$

The authors show that problem (19) can be expressed in terms of ordinary differential equations that need to be solved numerically. Kim et al. (2008) provide the first study investigating the stochastic control problem in a multivariate framework. They contribute to existing literature by providing computationally tractable solutions for the optimal portfolio holdings. However, transaction costs are not included in their problem formulation. For futher research, Kim et al. (2008) raise an interesting question - it should be evaluated whether the benefit from diversification across various spreads compensates losses due to unfavorable shocks that destabilize complete multivariate portfolios. Further investigation of optimal pairs trading with multiple pairs based on a mathematical programming approach can be found in Yamamoto and Hibiki (2017).

Chiu and Wong (2011) focus on optimal multivariate investment allocation over cointegrated assets, providing the first theoretical work in that context. They consider one risk-free asset and $n$ stocks, from which $N(1 \leq N \leq n)$ cointegrating vectors $z_{t}=\left\{z_{1, t}, \ldots, z_{N, t}\right\}$ are constructed:

$$
z_{j, t}=\alpha_{j}+\beta_{j} t+\sum_{i=1}^{n} c_{i j} \ln S_{i, t}, \quad j=1, \ldots, N
$$

where $S_{i, t}$ is the price of asset $i$ at time $\mathrm{t}$ for $i=1, \ldots, n$. The cointegrating vectors $z_{t}$ follow the multivariate OU process of equation (18). The optimal portfolio weights are determined by solving the following problem:

$$
\begin{aligned}
& \underset{b}{\min } \operatorname{Var}\left[V_{T}\right] \\
& \text { subject to } E\left[V_{T}\right]=v_{T}
\end{aligned}
$$

for portfolio weights $b$, terminal wealth $V_{T}$, and expected final wealth $v_{T}$. The authors provide closed-form solutions of problem (20). The approach by Chiu and Wong (2011) generalizes classic pairs trading in two respects. First, the cointegrating vector consists of $n$ risky assets instead of two. Second, the dollar-neutrality assumption, i.e., the assumption that the investor trades equal amounts in the long and short positions, is dropped since the coefficients $c_{i j}$ are determined 
separately for each stock. Further research should provide an empirical application with multiple spreads based on the multivariate theoretical framework. Lintilhac and Tourin (2017) consider a similar framework as Chiu and Wong (2011). Main difference lies in the objective function: Instead of minimizing the variance of terminal wealth as in (20), they maximize the expected utility from terminal wealth for an investor with exponential utility. Analytic results for the optimal portfolio weights are provided.

Chiu and Wong (2015) expand upon Chiu and Wong (2011) and investigate time-consistent portfolio selection with cointegration, providing an optimal timeconsistent asset allocation policy. Chiu and Wong (2018) study robust dynamic pairs trading with cointegration subject to parameter estimation errors.

To summarize the subsection on multivariate OU models, it should be pointed out that literature in this context is sparse due to analytic tractability issues (see Jurek and Yang 2007) ${ }^{10}$. Given the high practical relevance of multivariate modeling (see Meucci 2009), future research should aim at providing further analytic results in that context.

\subsection{Lévy driven Ornstein-Uhlenbeck model}

The normality assumption of the classic OU process is an obvious deficit since stock prices and returns exhibit fat tails and jumps (see, e.g., Cont 2001, Bertram 2009, Göncü and Akyildirim 2016a, Stübinger and Endres 2018). Replacing the Brownian motion $\left\{W_{t}\right\}_{t \geq 0}$ by a Lévy-process $\left\{L_{t}\right\}_{t \geq 0}$ leads a more flexible process:

$$
d X_{t}=\theta\left(\mu-X_{t}\right) d t+\sigma d L_{t}
$$

The model (21) includes as special cases (i) the classic OU process driven by Brownian motion, and (ii) a jump-diffusion model, in case the Lévy process $\left\{L_{t}\right\}_{t \geq 0}$ consists of Brownian diffusion and jumps caused by a Poisson process $\left\{N_{t}\right\}_{t \geq 0}$ with intensity $\lambda \geq 0$. Different variants of jump-diffusion models are applied in literature, e.g.,

$$
d X_{t}=\theta\left(\mu(t)-X_{t}\right) d t+\sigma d W_{t}+\ln J_{t} d N_{t}
$$

\footnotetext{
10 There are some studies (see, e.g., D'Aspremont 2011 and Cartea and Jaimungal 2016) that construct one mean-reverting portfolio $P_{t}$ consisting of multiple stocks $S_{1}, \ldots, S_{n}$. The mispricing in this case is of the form $P_{t}=\sum_{i=1}^{n} x_{i} S_{i}(t)$ with coefficients $x_{i}, i=1, \ldots, n$. These works are not covered by our definition of a multivariate framework since the underlying OU process for portfolio $P_{t}$ is still univariate.
} 
with a random variable $J_{t}$ modeling the jump sizes or

$$
d X_{t}=-\theta X_{t} d t+\sigma d W_{t}+d C_{t}
$$

with compound Poisson process $C_{t}=\sum_{k=1}^{N_{t}} J_{k}$ and a sequence of independent random variables $\left\{J_{k}\right\}_{k=1}^{\infty}$ with common symmetric density $\varphi$.

Table 3 summarizes the relevant works applying a Lévy driven OU model to capture the spread dynamics. To the present day, there exist only four academic studies in this context.

\subsubsection{Analytic approach}

Model-driven decisions. Stübinger and Endres (2018) develop a high-frequency pairs trading framework based on a mean-reverting jump-diffusion model. The spread $X_{t}$ follows the jump-diffusion model (22) with varying jump intensity $\lambda(t)$ such that

$$
\lambda(t)= \begin{cases}0 & \text { if the observation is intraday } \\ \lambda & \text { otherwise (overnight, weekend) }\end{cases}
$$

The authors verify the existence of overnight jumps in their empirical data set and therefore consider the jump component $\ln J_{t} d N_{t}$ in their model. In the formation period, pairs selection is optimized by choosing pairs with highest mean-reversion rate $\theta$ and highest jump intensity $\lambda$. A large mean-reversion rate ensures fast convergence to the equilibrium level $\mu(t)$, where pairs trading profits are taken. A high jump intensity creates sudden, large spread movements and thus many trading opportunities. In the trading period, thresholds vary around the mean-reversion level $\mu(t)$ of the process. The main contributions of this study are (i) the integration of a jump component into the classic mean-reverting model and (ii) the consideration of the model's mean-reverting patterns and jump behavior in both the formation and the trading period.

Optimal trading thresholds. Göncü and Akyildirim (2016a) as well as Endres and Stübinger (2019b) determine optimal trading thresholds in the sense of Bertram (2010b), hereby explaining the spread dynamics by the Lévy driven OU model of equation (21).

Göncü and Akyildirim (2016a) assume that the marginals of the Lévy process $\left\{L_{t}\right\}_{t \geq 0}$ follow the generalized hyperbolic distribution. The authors consider three cases of pairs trading strategies. We summarize them in one framework as follows. A trader opens a position when the spread crosses threshold $a$ and closes the position at threshold $m, a<m$. 
At any time $T$, the return from this trade is

$$
r(a, m, T)=\left\{\begin{array}{lc}
m-a, & \tau_{m, a} \leq T \\
X_{T}-a, & \tau_{m, a}>T
\end{array}\right.
$$

with $\tau_{m, a}=\inf \left\{t>0 \mid X_{t}=m, X_{0}=a\right\}$. The investor maximizes the expected value of these trading profits

$$
\max _{a, m}\left\{P\left(\tau_{m, a}<T\right)(m-a)+\left(1-P\left(\tau_{m, a}<T\right)\right) E\left[X_{T}-a \mid \tau_{m, a}>T\right]\right\}
$$

The authors estimate the first passage time probability $P\left(\tau_{m, a}<T\right)$ via Monte Carlo simulation since there is no closed form solution for the first passage time density of the Lévy driven OU process available. Based on these Monte Carlo estimations, they solve problem (25) and provide trading signals in form of profit maximizing thresholds. In contrast to the baseline approach by Bertram (2010b), the authors extend the objective function by considering the probability of the spread's divergence (see equation 24). However, the authors do not consider transaction costs in their strategy.

Endres and Stübinger (2019b) model the spread dynamics according to equation (21). An investor opens positions at threshold $a$ and closes at $m, a<m$. The authors follow Bertram (2010b) and maximize the expected return per unit of time in their strategy

$$
\max _{a, m} \frac{r(a, m, c)}{E\left[\tau_{m, a}\right]}
$$

for return $r(a, m, c)=m-a-c$ and transaction costs $c$. Decomposing the process $\left\{L_{t}\right\}_{t \geq 0}$ into two parts $-\left\{Q_{t}\right\}_{t \geq 0}$, representing the downward jumps and the Brownian motion, and $\left\{\boldsymbol{R}_{t}\right\}_{t \geq 0}$, representing the upward jumps - there is an explicit representation of the expected first-passage time $E\left[\tau_{m, a}\right]$ available. Based on this result, problem (26) can be solved directly and the optimal trading bounds $a$ and $m$ are obtained. In contrast to Göncü and Akyildirim (2016a), no Monte Carlo methods are needed for solving the optimization problem.

\subsubsection{Optimal timing of trades - dynamic programming}

Larsson et al. (2013) study the problem of optimally closing a pairs trade when the spread $X_{t}$ is modeled by an OU process extended with a jump component (see equation 23). Assuming the investor has already opened a position in the spread, the authors consider the following optimal stopping problem

$$
V(x)=\sup _{\tau \leq \tau_{a}} E\left[X_{\tau}\right]
$$


for a stop-loss level $a<0$ and $\tau_{a}=\inf \left\{t \geq 0: X_{t} \leq a\right\}$. In case the spread falls below $a$, the position is closed in order to limit potential losses. Applying stochastic control theory, the authors analyze a numerical method for solving the free boundary problem (27). In the context of Lévy driven OU models, Larsson et al. (2013) provide the first study dealing with a stochastic control problem in pairs trading. The incorporation of stop-loss levels reduces the risk of model misspecification and potential failure of the optimal stopping problem (see Yoshikawa 2017).

To summarize the subsection on Lévy driven OU models, it should be pointed out that analytic formulas have not yet been derived in this context. Since the process has recently attracted attention, e.g., due to the presence of jumps in high-frequency data (see Jondeau et al. 2015), future work should build upon the aforementioned studies and provide further closed-form results.

\subsection{Regime-switching Ornstein-Uhlenbeck model}

Pairs trading strategies typically rely on the spread's convergence to a specific mean. Switches of this mean between different levels lead to failure of traditional trading approaches in practice (see Bock and Mestel 2009). Speaking more generally, it is unrealistic to assume constant model parameters in the long run. This drawback is eliminated by regime-switching models - they consider the change of parameters over time based on the theory of Markov processes. The spread in the regime-switching OU model follows

$$
d X_{t}= \begin{cases}\theta_{1}\left(\mu_{1}-X_{t}\right) d t+\sigma_{1} d W_{t}, & \text { for } R_{t}=1 \\ \vdots & \vdots \\ \theta_{r}\left(\mu_{r}-X_{t}\right) d t+\sigma_{r} d W_{t}, & \text { for } R_{t}=r\end{cases}
$$

and switches between $r$ different regimes $(r \in \mathbb{N})$. The continuous-time Markov chain $\left\{R_{t}\right\}_{t \geq 0}$ describes the regime switching behavior and allows for $r$ different states. The random variable $R_{t}$ denotes the state of the process at time $t^{11}$.

Table 3 provides an overview of the studies applying a regime-switching OU model to capture the spread dynamics. To date, there exist only four works in that context.

\subsubsection{Optimal trading thresholds - analytic approach}

Bai and Wu (2018) address an optimal investment problem based on a regimeswitching OU model with $r$ different states. The spread $X_{t}$ evolves according to

${ }^{11}$ In the special case of only one state, i.e., $r=1$, the regime-switching model (28) reduces to the classic OU process. 
equation (28). The authors give a closed-form expression for the pairs trading value function, i.e., the expected return per unit of time. This is basically the same objective function as in Bertram (2010b) (see equation 6), with the difference that $r$ possible states are considered. The authors derive analytic solutions for systems of ordinary differential equations and obtain an optimum of the value function with trading thresholds $a$ and $m, a<m$. A trade is entered, when the spread $X_{t}$ crosses one of the thresholds. However, explicit analytic solutions are only presented for $r=1$, i.e., one regime, which is the case without any switching already considered in Bertram (2010b). Further, the numerical analysis is executed only for one-state and two-state regime switching models. For future research, it would be interesting to see how the approach works for more than two regimes. Moreover, as the authors themselves point out, the pairs trading rule is static - further research could construct dynamic optimal thresholds that are more suitable for practical applications.

\subsubsection{Optimal investment allocation - dynamic programming}

Altay et al. (2017) focus on dynamic portfolio optimization and derive optimal portfolio holdings for an investor with logarithmic utility. The spread $X_{t}$ is modeled by an OU process with Markov modulated mean-reversion level, i.e., the mean level switches across $r(r \in \mathbb{N})$ different regimes. For a dollar-neutral portfolio of two stocks with weights $b_{1}(t)=-b_{2}(t)$, the authors maximize the expected utility from terminal wealth, penalized by the riskiness of the portfolio. The optimal weight is derived via pointwise maximization as

$$
b_{1}^{*}(t, x, i)=\frac{1}{1+\varepsilon}\left(\frac{\theta\left(\mu_{i}-x\right)}{\sigma^{2}}+\frac{\rho \eta}{\sigma}-\frac{1}{2}\right), \quad i \in\{1, \ldots, r\}
$$

for risk factor $\varepsilon>0$, correlation $\rho$, and volatility $\eta$ of stock 1 . It is an interesting approach to prevent the trader from taking risky positions by penalizing the objective function by the realized volatility. Further, the important problem of a constant mean level is addressed, which is unrealistic in practice but still assumed by many studies (see Bock and Mestel 2009). However, there is one main downside associated with the model: In contrast to Bai and Wu (2018), only the mean level is allowed to switch between the different regimes - all other model parameters, i.e., mean-reversion speed and volatility, remain constant.

\subsubsection{Other approaches}

This subsection discusses other approaches based on the regime-switching OU model beyond analytic and dynamic programming frameworks.

Model-based trading rules. Yang et al. (2016) assume that the spread follows equation (28) and is divided into two states: $R_{t}=$ "high" and $R_{t}=$ "low". All model 
parameters, i.e., $\mu, \theta$, and $\sigma$, vary across the two regimes. In the formation period, the authors select pairs by smallest sum of squared differences. At this point, a simple yet promising improvement would be to optimize pairs selection by choosing pairs based on model parameters instead of simple distance metrics. In the trading period, three rules are implemented - two classic benchmark rules and one rule based on the regime-switching model. In the latter case, the spread value is predicted one step ahead from the calibrated model - if it deviates by more than 1.96 standard deviations from its observed value, a position is opened. The critical value of $\mathbf{1 . 9 6}$ is chosen in accordance with the $95 \%$ confidence interval for the normal distribution.

Regime classification algoritbm. Endres and Stübinger (2019a) develop a regime-switching framework for Lévy driven OU models with different regimes. They specify the spread $X_{t}$ by equation (28), providing the first study that replaces the Brownian motion by the Lévy process $\left\{L_{t}\right\}_{t \geq 0}$ in a regime-switching framework. As such, the model is capable of explaining fat tails and jumps within the individual regimes. All model parameters are allowed to vary across the states. Hereby, the number of states is not determined in advance. The authors develop a regime classification algorithm that allows the process to switch between a flexible number of regimes. This is in contrast with Yang et al. (2016), who assume $r=2$ regimes in their study, as well as Altay et al. (2017) and Bai and Wu (2018), who assume a fixed number of regimes.

To summarize, relatively few studies consider the change of parameters over time based on regime-switching models. It should be investigated whether switches of parameters are recognized quickly enough in these models to appropriately adjust the trading rules (see Bogomolov 2013).

\section{Advanced mean-reverting diffusion models}

Mean-reverting diffusion models beyond the OU process are Cox-IngersollRoss (CIR), inhomogeneous geometric Browian motion (IGBM), and stochastic volatility (SV) models. These models have a drift term identical to the OU process, while the diffusion term is extended - as such, the disadvantage of constant volatility is overcome (see, e.g., Stehlıková 2008, Chanol et al. 2015). The relevant studies are summarized in Table 4.

\subsection{Cox-Ingersoll-Ross model}

The CIR process overcomes the constant volatility assumption of the OU process, and still exhibits the important mean-reverting property. However in this model, analytic calculations become more challenging. 
The spread follows

$$
d X_{t}=\theta\left(\mu-X_{t}\right) d t+\sigma \sqrt{X} d W_{t}
$$

with parameters $\mu, \theta, \sigma>0$ and standard Brownian motion $\left\{W_{t}\right\}_{t \geq 0}$. The diffusion term $\sigma \sqrt{X}_{t}$ is extended compared to the OU process. In case the process approaches zero, the mean-reverting drift term dominates the decreasing diffusion term, ensuring nonnegativity of the process values. However, Avonleghi and Davison (2017) point out that negative price spreads are an essential modeling feature - the non-negativity assumption actually stems from modeling interest rates, volatility, and prices (see, e.g., Heston 1993) rather than spreads. In practice, the disadvantage of non-negativity might present no issue if the long-term mean of the spread is far enough from zero.

Table 4

Advanced mean-reverting diffusion models

\begin{tabular}{|l|l|}
\hline \multicolumn{1}{|c|}{ Model Cox-Ingersoll-Ross model } \\
\hline \multicolumn{2}{|c|}{ Analytic approach } \\
\hline \multicolumn{2}{|c|}{ Dynamic programming } \\
\hline $\begin{array}{l}\text { Optimal trading thresholds: Maximum expected } \\
\text { return and Sharpe ratio per unit of time }\end{array}$ & Gregory et al. (2010) \\
\hline \multicolumn{2}{|c|}{ Dynamic programming } \\
\hline $\begin{array}{l}\text { Optimal timing of trades: Optimal starting- } \\
\text { stopping problem, finite and infinite trading } \\
\text { horizons }\end{array}$ & Li (2015), Leung and Li (2016), \\
\hline \multicolumn{2}{|c|}{ Kitapbayev and Leung (2018) } \\
\hline \multicolumn{2}{|c|}{ Analytic approach } \\
\hline $\begin{array}{l}\text { Optimal timing of trades: Maximum reward } \\
\text { function, optimal switching problem }\end{array}$ & and Leung (2018) \\
\hline \multicolumn{2}{|c|}{ Dynamic programming } \\
\hline \multicolumn{2}{|c|}{ Inhomogeneous geometric Brownian motion model } \\
\hline $\begin{array}{l}\text { Optimal trading thresholds: Maximum expected } \\
\text { return and Sharpe ratio per unit of time }\end{array}$ & $\begin{array}{l}\text { Ngo and Pham (2016), Kitapbayev } \\
\text { and Leung (2018) }\end{array}$ \\
\hline \multicolumn{2}{|c|}{ Optimal timing of trades: Maximum reward } \\
function, optimal trading boundaries
\end{tabular}




\subsubsection{Optimal trading thresholds - analytic approach}

Gregory et al. (2010) model spread and ratio dynamics according to the CIR model of equation (29). Following Bertram (2010b), the expected trade return $\mu(a, m, c)$ and the variance of trade returns $\sigma^{2}(a, m, c)$ are determined by equations (6) and (8). With the aid of first passage time results for CIR processes, this leads

$$
\left.\mu(a, m, c)=r(a, m, c) /\left\{\begin{array}{l}
\left.\frac{d \Phi}{d a_{n}}\left(0, \frac{2 \theta \mu}{\sigma^{2}}, \frac{2 \theta}{\sigma^{2}} a\right)-\frac{d \Phi}{d a_{n}}\left(0, \frac{2 \theta \mu}{\sigma^{2}}, \frac{2 \theta}{\sigma^{2}} m\right)+\right] \\
\frac{d \Psi}{d a_{n}}\left(0, \frac{2 \theta \mu}{\sigma^{2}}, \frac{2 \theta}{\sigma^{2}} m\right)-\frac{d \Psi}{d a_{n}}\left(0, \frac{2 \theta \mu}{\sigma^{2}}, \frac{2 \theta}{\sigma^{2}} a\right)
\end{array}\right]\right\}
$$

and a return variance $\sigma^{2}(a, m, c)$ derived similarly. $\Phi\left(a_{n}, b, z\right)$ and $\Psi\left(a_{n}, b, z\right)$ denote the Kummer's and Trinomi function (see Zhao 2009). For the spread $X_{t}=\ln S_{1}(t)-\ln S_{2}(t)$, the trade return is $r(a, m, c)=m-a-c$ and for the ratio $\tilde{X}_{t}=\frac{S_{1}(t)}{S_{2}(t)}$ it is $\tilde{r}(a, m, c)=\left(e^{m}-e^{a}-c\right)$. From these expressions, the authors determine the optimal trading strategy by maximizing expected return and Sharpe ratio numerically, obtaining the optimum trade levels $a$ and $m$. The construction has one major advantage: Traditional rules of thumb are replaced by optimal trading signals. However, and in contrast to subsection 2.1.2, no closed-form expressions for the thresholds $a$ and $m$ are provided. From an empirical perspective, it would be interesting to discuss the non-negativity assumption and suitability of the CIR process for spread modeling.

\subsubsection{Optimal timing of trades - dynamic programming}

Li (2015), Leung and Li (2016), and Kitapbayev and Leung (2018) analyze the optimal timing to open and subsequently close pairs trading positions. Capital is allocated to a mean-reverting portfolio evolving according to a CIR process (see equation 29) or a risk-free asset with rate $r>0$. For an investor who already holds a position $X_{t}$, e.g., one long and one short asset, the aim is to maximize the position's expected discounted value. As such, the investor solves the following optimal stopping problem:

$$
V(x)=\sup _{\tau \in \mathrm{T}} E\left[e^{-r \tau}\left(X_{\mathrm{T}}-c_{\text {sell }}\right)\right]
$$

If the position is closed at $\tau$, the investor receives the value $X_{\tau}$ and pays transaction costs $c_{\text {sell }}>0$. The entry timing is determined in the optimal starting-stopping problem:

$$
J(x)=\sup _{v \in \mathrm{T}} E\left[e^{-r v}\left(V\left(X_{v}\right)-X_{v}-c_{b u y}\right)\right]
$$


If the position is opened at time $v$, the investor pays transaction costs $c_{b u y}>0$ plus the process value $X_{v}$ and receives the expected value $V\left(X_{v}\right)$ from optimally stopping the process. Li (2015) and Leung and $\mathrm{Li}$ (2016) give analytic expressions for the value functions (31) and (32) with regard to hypergeometric functions and characterize the corresponding optimal trading boundaries. In contrast to the majority of studies, the transaction costs $c_{b u y}$ and $c_{\text {sell }}$ for entry and exit are allowed to differ. However, the trader has an unlimited amount of time for opening and closing positions. In practice, it might not be feasible that positions are held infinitely. This issue is only partly addressed by discounting the portfolio value with the risk-free rate $r$. In this respect, Kitapbayev and Leung (2018) enhance problems (31) and (32) for finite deadlines to enter and exit the market. However and in contrast to problems with infinite trading horizon, closed-form solutions cannot be derived.

\subsection{Stochastic volatility model}

In the stochastic volatility (SV) model, the spread $X_{t}$ follows

$$
d X_{t}=\theta\left(\mu-X_{t}\right) d t+\sigma\left(t, X_{t}\right) d W_{t}
$$

with a general diffusion term $\sigma\left(t, X_{t}\right)$. The SV model is popular because of its flexibility, resolving the issue of constant volatility of the OU model and enabling better matching to real world data (see, e.g., Ackerer et al. 2018). Further, the model includes prominent special cases, i.e., the OU model for $\sigma\left(t, X_{t}\right)=\sigma$, the CIR model for $\sigma\left(t, X_{t}\right)=\sigma \sqrt{X}_{t}$, and the IGBM model for $\sigma\left(t, X_{t}\right)=\sigma X_{t}$. Table 4 provides an overview of the relevant studies using a SV model to capture the spread dynamics. As opposed to the previous sections, no analytic approach has been published.

Ngo and Pham (2016) explain the spread $X_{t}$ by the mean-reverting stochastic volatility model (see equation 33). Pairs trading is considered as a switching problem between three regimes: no holding of stocks $(i=0)$, one stock long the other short $(i=1)$, and vice-versa $(i=-1)$. The trading strategy is modeled by $\alpha=\left(\tau_{n}, \mathrm{t}_{n}\right)_{n \geq 0}$, consisting of a sequence of trading times $\left(\tau_{n}\right)_{n}$ and $\mathrm{t}_{n} \in\{-1,0,1\}$ representing the flat, long, or short position. The investor maximizes the expected discounted cumulative gain penalized by the holding of assets over an infinite horizon:

$$
v(x)=\sup _{\alpha} E\left[\sum_{n \geq 1} e^{-\rho \tau_{n}} g\left(X_{\tau_{n}}, \alpha_{\tau_{n}^{-}}, \alpha_{\tau_{n}}\right)-\lambda \int_{0}^{\infty} e^{-\rho \tau_{n}}\left|\alpha_{\tau}\right| d t\right]
$$

for gain function $g$, discount factor $\rho>0$, initial spread value $X_{0}=x$, and penalizing factor $\lambda \geq 0$. The authors are able to directly derive the structure of the 
switching regions and the form of the value function $v$ via partial differential equations. Hereby, the solutions $\psi_{+}$and $\psi_{-}$of the following system need to be determined

$$
\begin{aligned}
& \mathfrak{L} \varphi(x)=\theta(\mu-x) \varphi^{\prime}(x)+\frac{1}{2} \sigma^{2}(x) \varphi^{\prime \prime}(x) \\
& \rho \phi-\mathfrak{L} \phi=0
\end{aligned}
$$

The solutions of (35) specify the switching regions where trading decisions are executed. Interestingly and in contrast to the majority of studies, the optimization problem (34) is extended for no holding of stocks in addition to classic long and short positions. Hereby, the flat position is explicitly penalized with a factor $\lambda$. Kitapbayev and Leung (2018) consider a similar problem as Ngo and Pham (2016). The two main differences are (i) Ngo and Pham (2016) focus on cumulated rewards gained by multiple trades, Kitapbayev and Leung (2018) consider the discounted reward of one trade cycle, (ii) Ngo and Pham (2016) consider a problem over infinite horizon, Kitapbayev and Leung (2018) integrate a finite deadline to enter and exit the market - however, this deadline does not allow for closed-form solutions any more.

\subsection{Inhomogeneous geometric Brownian motion model}

In the mean-reverting IGBM model (also called GARCH diffusion, GBM with affine drift), the spread $X_{t}$ evolves according to

$$
d X_{t}=\theta\left(\mu-X_{t}\right) d t+\sigma X_{t} d W_{t}
$$

The diffusion term $\sigma X_{t}$ is non-constant and depends on the process value itself. Increasing $X_{t}$ leads to an increasing volatility. The model is a special case of the stochastic volatility model (see equation 33). Table 4 summarizes the relevant works applying an IGBM model to explain the spread dynamics.

\subsubsection{Optimal trading thresholds - analytic approach}

Gregory et al. (2010) model spread and ratio dynamics by the IGBM model (see equation 36). As described in subsection 4.1.1 for the CIR process, the authors follow Bertram (2010b) and derive closed-form expressions for two pairs trading objective functions - expected return $\mu(a, m, c)$ and Sharpe ratio $S\left(a, m, c, r_{f}\right)$ of the strategy. 
It is

$$
\begin{aligned}
& \mu(a, m, c)= \\
& =\frac{r(a, m, c)}{2\left(2 \theta+\sigma^{2}\right)}\left[\begin{array}{l}
\ln \frac{m}{a}+\ln \frac{a}{m}+\frac{d \Phi}{d a_{n}}\left(0,2+\frac{2 \theta}{\sigma^{2}}, \frac{2 \theta \mu}{\sigma^{2} a}\right)- \\
\frac{d \Phi}{d a_{n}}\left(0,2+\frac{2 \theta}{\sigma^{2}}, \frac{2 \theta \mu}{\sigma^{2} m}\right)+\frac{d \Psi}{d a_{n}}\left(0,2+\frac{2 \theta}{\sigma^{2}}, \frac{2 \theta \mu}{\sigma^{2} m}\right)-\frac{d \Psi}{d a_{n}}\left(0,2+\frac{2 \theta}{\sigma^{2}}, \frac{2 \theta \mu}{\sigma^{2} a}\right)
\end{array}\right]
\end{aligned}
$$

and $S\left(a, m, c, r_{f}\right)$ similarly. These objective function are maximized numerically to obtain the optimum entry and exit bounds $a$ and $m$. Direct optimization via differentiation has only been accomplished for the classic OU process in Bertram (2010b) yet.

\subsubsection{Optimal timing of trades - dynamic programming}

Ngo and Pham (2016) explain the spread dynamics $X_{t}$ by the IGBM model (see equation 36). The authors identify optimal trading boundaries for a pairs trading strategy that aims at maximizing the expected reward (see equation 34). The two fundamental solutions for problem (35) in case of the IGBM model are given by

$$
\psi_{+}(x)=x^{-a} \Psi\left(a, b, \frac{c}{x}\right), \quad \psi_{-}(x)=x^{-a} \Phi\left(a, b, \frac{c}{x}\right)
$$

with $\Phi$ and $\Psi$ the confluent hypergeometric function of first and second kind and

$$
a=\frac{\sqrt{\sigma^{2}+4(\theta+2 \rho) \sigma^{2}+4 \theta^{2}}-\left(2 \theta+\sigma^{2}\right)}{2 \sigma^{2}}, b=\frac{2 \theta}{\sigma^{2}}+2 a+2, c=\frac{2 \theta \mu}{\sigma^{2}}
$$

Based on these solutions, the structure of the switching regions for the IGBM model is directly specified. Kitapbayev and Leung (2018) consider a similar problem - compared to the case of a stochastic volatility model (see subsection 4.2), the optimal trading boundary problem simplifies for the special case of the IGBM model.

To summarize the section on advanced mean-reverting diffusion models, there remains a lack of studies that investigate the additional benefits of the CIR, IGBM, and SV models in pairs trading. It has not been clearly studied how spread modeling as well as pairs selection and trading can be improved with these models.

\section{Diffusion models with a non-stationary component}

The vast majority of studies models the spread as a stationary mean-reverting process. However, spreads may also exhibit non-stationary effects (Bertram 2009, 
Bertram 2010a, Focardi et al. 2016, Tie et al. 2017). As such, models based on stationary time series are not suitable for reliable forecasting and decision making (Bertram 2010a). Besides extending the classic OU process by jumps (see subsection 3.2) and regime-switches (see subsection 3.3), there exist other possibilities to incorporate non-stationarity, which are summarized in Table 5 and discussed in the following subsections.

Table 5

Diffusion models with a non-stationary component

\begin{tabular}{|c|c|}
\hline Model & Study \\
\hline \multicolumn{2}{|c|}{ 5.1. Geometric Brownian motion model } \\
\hline \multicolumn{2}{|c|}{ Dynamic programming } \\
\hline $\begin{array}{l}\text { Optimal timing of trades: Sequential buying } \\
\text { and selling times determined by threshold } \\
\text { curves }\end{array}$ & Tie et al. (2017) \\
\hline $\begin{array}{l}\text { Optimal investment allocation: Optimal } \\
\text { portfolio holdings under parameter ambiguity }\end{array}$ & Fouque et al. (2016) \\
\hline \multicolumn{2}{|c|}{ 5.2. Two-factor model } \\
\hline \multicolumn{2}{|c|}{ Analytic approach } \\
\hline $\begin{array}{l}\text { Optimal trading thresholds: Maximum } \\
\text { expected return and Sharpe ratio per unit of } \\
\text { time }\end{array}$ & $\begin{array}{l}\text { Bertram (2010a), Gu and Steffensen } \\
(2015)\end{array}$ \\
\hline \multicolumn{2}{|c|}{ 5.3. General Itô diffusion model } \\
\hline \multicolumn{2}{|c|}{ Analytic approach } \\
\hline $\begin{array}{l}\text { Optimal trading thresholds: Maximum } \\
\text { expected rate of profit under drawdown } \\
\text { constraint }\end{array}$ & Bertram (2009) \\
\hline
\end{tabular}

\subsection{Geometric Brownian motion model}

According to Tie et al. (2017), limiting research to mean-reverting spreads adds a strong restriction on pairs trading strategies and their application. To fulfill the mean-reversion requirement, matching of stocks is typically executed among assets of the same industry. From a practical point of view, there is a broader range of potential pairs with desirable properties beyond the mean-reverting pairs. Instead of classic spread modeling, two stocks can be modeled separately 
by geometric Brownian motions (GBM), dropping the typical mean-reversion requirement. The risky assets with prices $S_{1}(t)$ and $S_{2}(t)$ follow

$$
\left(\begin{array}{l}
d S_{1}(t) \\
d S_{2}(t)
\end{array}\right)=\left(\begin{array}{cc}
S_{1}(t) & 0 \\
0 & S_{2}(t)
\end{array}\right)\left[\left(\begin{array}{l}
\mu_{1} d t \\
\mu_{2} d t
\end{array}\right)+\left(\begin{array}{ll}
\sigma_{11} & \sigma_{12} \\
\sigma_{21} & \sigma_{22}
\end{array}\right)\left(\begin{array}{l}
d W_{1}(t) \\
d W_{2}(t)
\end{array}\right)\right]
$$

with two-dimensional standard Brownian motion $W(t)=\left(W_{1}(t), W_{2}(t)\right)$. The correlation between $S_{1}(t)$ and $S_{2}(t)$ is regulated by $\sigma_{12}=\sigma_{21}$. In other notation, the risky assets follow

$$
d S_{i}(t)=\mu_{i} S_{i}(t) d t+\sigma_{i} S_{i}(t) d W_{i}(t), i=1,2
$$

with $W(t)=\left(W_{1}(t), W_{2}(t)\right)$ and $d W_{1}(t) d W_{2}(t)=\rho d t, \rho \in[-1,1]$.

Table 5 provides an overview of the studies applying a GBM model to explain the spread dynamics. As opposed to the previous sections, no analytic approach has been published.

\subsubsection{Dynamic programming}

Optimal timing of trades. Tie et al. (2017) explain the stock prices $S_{1}(t)$ and $S_{2}(t)$ based on GBM models as specified in equation (38). In their pairs trading strategy, the authors maximize an overall return by sequential and simultaneous trading of stock pairs. It is assumed that the initial position in the spread $X_{t}=S_{1}(t)-S_{2}(t)$ can either be flat $(i=0)$ or long $(i=1)$. For $\tau_{0} \leq \tau_{1} \leq \tau_{2} \leq \ldots$, pairs are sold at stopping times $\tau_{0}, \tau_{2}, \ldots$ and bought at $\tau_{1}, \tau_{3}, \ldots$ respectively. A trading sequence is denoted by $\Lambda_{0}=\left(\tau_{1}, \tau_{2}, \ldots\right)$ for $i=0$ and $\Lambda_{1}=\left(\tau_{0}, \tau_{1}, \tau_{2}, \ldots\right)$ for $i=1$. For transaction costs $c$, initial position $i=0$, discount factor $\rho>0, c_{b}=1+c$, and $c_{s}=1-c$, the reward function $J_{0}\left(s_{1}, s_{2}, \Lambda_{0}\right)$ is

$$
\begin{aligned}
& E\left[e^{-\rho \tau_{2}}\left(c_{s} S_{1}\left(\tau_{2}\right)-c_{b} S_{2}\left(\tau_{2}\right)\right) 1_{\left\{\tau_{2}<\infty\right\}}-e^{-\rho \tau_{1}}\left(c_{b} S_{1}\left(\tau_{1}\right)-c_{s} S_{2}\left(\tau_{1}\right)\right) 1_{\left\{\tau_{1}<\infty\right\}}\right. \\
& \left.+e^{-\rho \tau_{4}}\left(c_{s} S_{1}\left(\tau_{4}\right)-c_{b} S_{2}\left(\tau_{4}\right)\right) 1_{\left\{\tau_{4}<\infty\right\}}-e^{-\rho \tau_{3}}\left(c_{b} S_{1}\left(\tau_{3}\right)-c_{s} S_{2}\left(\tau_{3}\right)\right) 1_{\left\{\tau_{3}<\infty\right\}}+\ldots\right]
\end{aligned}
$$

and for $i=1$ it is $J_{1}\left(s_{1}, s_{2}, \Lambda_{1}\right)$ calculated similarly. The investor solves the optimal control problem

$$
V_{i}\left(s_{1}, s_{2}\right)=\sup _{\Lambda_{i}} J_{i}\left(s_{1}, s_{2}, \Lambda_{i}\right), \quad i=0,1
$$

The authors characterize the optimal policy, i.e., the optimal entry and exit times, by threshold curves obtained with dynamic programming techniques. These curves constitute three regions: selling zone $\Gamma_{1}$, holding zone $\Gamma_{2}$, and buying zone $\Gamma_{3}$. A larger correlation between stocks 1 and 2, measured by the 
parameter $\sigma_{12}$, leads to greater buying and selling zones and thus more trading opportunities. Tie et al. (2017) provide a clear value-add to pairs trading research - the authors relax the the typical mean-reversion requirement of the spread. Thus, the model includes a broader range of potential pairs with desirable properties - among them still the classic mean-reverting pairs. From a practical perspective, this offers wider selection possibilities and new opportunities in pairs trading. However, there is a downside associated with the GBM model to explain the spread dynamics: As in the classic OU model, the assumption of constant volatility or correlation is not always appropriate (see, e.g., Mota and Esquivel 2016, Avonleghi and Davison 2017). For futher research, Tie et al. (2017) suggest to use more realistic models for stock dynamics, e.g., extend the GBMs with regime switching ${ }^{12}$.

Optimal investment allocation. Fouque et al. (2016) study an investment allocation problem, in which an investor trades a risk-free asset $M_{t}$ or two risky assets. The amounts invested in the assets are denoted by $\boldsymbol{b}_{i}(t), i=0,1,2$. The two risky assets follow the GBM model (see equation 39) with ambiguous correlation $\rho_{t}$. The wealth of the investor is defined as $X_{t}=b_{0}(t)+b_{1}(t)+b_{2}(t)$ and evolves according to

$$
d X_{s}=\left[r X_{s}+\beta^{\prime} h_{s}\right] d t+\left[\sigma_{1} h_{1}(s) \sigma_{2} h_{2}(s)\right] d W_{s}, X_{t}=x
$$

for interest rate $r, b=\left[b_{1} b_{2}\right]^{\prime}$, and excess return vector $\beta$. The investor maximizes the expected utility in the worst-case scenario

$$
\underset{\Lambda_{i}}{\sup } \inf _{\rho} E\left[u\left(X_{T}\right) \mid X_{t}=x\right]
$$

The authors derive an analytic solution of problem (40) including closedform solutions for power and exponential utility. Depending on the covariance matrices between the two risky assets and the corresponding variance risk ratios, the market is in favor of classic pairs trading or directional trading. Hereby, the portfolio selection is robust to the uncertain correlation. In case of low correlation between the risky assets, spread trading is not optimal. This is in line with the findings of Tie et al. (2017) - higher correlation encourages greater pairs trading activity. Fouque et al. (2016) extend their approach to stochastic volatility models with ambiguous correlation and derive an asymptotic closed-form solution. The authors provide new directions for continuous-time pairs trading in three respects.

\footnotetext{
12 Göncü and Akyildirim (2014), Göncü (2015), and Göncü and Akyildirim (2017) analyze the existence of statistical arbitrage based in GBM models for single stocks with extensions for jumps and multi-asset frameworks. Their results could be extended for spreads, i.e., portfolios of two stocks, one long and one short.
} 
First, they present the first academic study that considers parameter ambiguity in their model. Second, they apply a trading strategy that comprises not only classic pairs trading but also other trading variants. Third, and in contrast to Tie et al. (2017), they extend their problem to stochastic volatility models, hereby resolving the issue of constant volatility in the GBM model.

\subsection{Two-factor model}

According to several studies (see, e.g., Schwartz 1997, Schwartz and Smith 2000, Farkas et al. 2017), more than one factor is necessary to describe uncertain price dynamics appropriately. The two-factor model in pairs trading has been proposed by Bertram (2010a). The spread $X_{t}$ is modeled as the sum of a stationary component $Y_{t}$, driving mean-reversion effects in the short-run, and a non-stationary component $M_{t}$, representing the long-term behavior. Hereby, $Y_{t}$ is modeled by an OU process, and $M_{t}$ by an arithmetic Brownian motion. The spread follows

$$
\begin{aligned}
& X_{t}=Y_{t}+M_{t} \\
& d Y_{t}=-\theta Y_{t} d t+\sigma d W_{t} \\
& d M_{t}=\gamma d t+\eta d Z_{t}
\end{aligned}
$$

with Brownian motions $\left\{W_{t}\right\}_{t \geq 0}$ and $\left\{Z_{t}\right\}_{t \geq 0}$. This non-stationary model allows for mean-reversion of the spread around a stochastic mean level. Table 5 summarizes the relevant works applying a two-factor model to capture the spread dynamics. Only two studies in this context have been published yet.

They key work is provided by Bertram (2010a) - the spread $X_{t}$ is explained by the two- factor model of equation (41). The author derives the optimal analytic pairs trading strategy under the framework described in subsection 2.1.2, i.e., trades are entered and exited when the spread crosses trading thresholds $a$ and $m, a<m$. For zero drift, i.e., $\gamma=0$, and independent Browian motions $\left\{W_{t}\right\}_{t \geq 0}$ and $\left\{Z_{t}\right\}_{t \geq 0}$, the author calculates analytic solutions for the expected return $\mu(a, m, c)$, variance $\sigma^{2}(a, m, c)$, and Sharpe ratio $S(a, m, c)$ of the strategy. The obtained results are almost identical with those for the classic OU process. The return is derived as

$$
\mu(a, m, c)=\frac{2 \theta(m-a-c)}{\pi\left(\operatorname{Erfi}\left(\frac{m \sqrt{\theta}}{\sigma}\right)-\operatorname{Erfi}\left(\frac{a \sqrt{\theta}}{\sigma}\right)\right)}
$$

and only differs from $\mu(a, m, c)$ for the OU model (equation 9) in a factor of 2. The only difference in the variance $\sigma^{2}(a, m, c)$ compared to the classic OU model 
is an additive term. In empirical examples, the author analyzes the influence of the non-stationary noise, represented by the parameter $\eta$, on the trading strategy. When maximizing the expected return, the non-stationary behavior does not have any influence on the thresholds. When maximizing the Sharpe ratio, increasing $\eta$ leads to strongly widened optimal trading bands - the strategy requires a higher return per trade to compensate the increasing variance. These results emphasize two major aspects. First, the trading strategy is strongly affected by non- stationary behavior. Second, the choice of a suitable objective function is highly important. Summarizing, the model by Bertram (2010a) provides a novelty in continuous-time pairs trading - it has the advantage that non-stationary effects can be captured and the model still exhibits mean-reverting behavior. However, the risk-free rate $r_{f}$ in the Sharpe ratio is assumed to be zero. Further, the author assumes zero drift and uncorrelated Brownian motions when deriving analytic formulas - as such, the model properties reduce to those of the classic OU process. It would be interesting to consider the model with non-zero correlation as it is studied for commodities in Schwartz and Smith (2000). Gu and Steffensen (2015) also propose a two-factor model, where the spread reverts to a stochastic mean level $\mu(t)$. However, the authors do not further specify trading rules based on this model.

\subsection{General Itô diffusion model}

In the general Itô diffusion model, the spread follows

$$
d X_{t}=\mu\left(t, X_{t}\right) d t+\sigma\left(t, X_{t}\right) d W_{t}
$$

with drift term $\mu\left(t, X_{t}\right)$ and diffusion term $\sigma\left(t, X_{t}\right)$. In this model, the spread is not necessarily mean-reverting. The powerful stochastic differential equation (43) incorporates the majority of stochastic spread models as special cases, i.e., classic OU, CIR, SV, IGBM, GBM, nonlinear mean-reverting, and skew mean-reverting model. To the present day, the only study considering a general Itô diffusion model to explain the spread dynamics is provided by Bertram (2009) (see Table 5).

Bertram (2009) examines the construction of an optimal analytic trading strategy under a drawdown constraint when the spread follows a general Itô diffusion process (see equation 43). Positions are entered and exited when the spread crosses the boundaries $a$ and $m, a<m-$ an analog construction is described in subsection 2.1 for the classic OU process. The total trade time $\tau$ is the time $\tau_{1}$ taken from entry to exit plus the time $\tau_{2}$ taken to enter a new trade. The optimal strategy is determined by maximizing the expected rate of profit

$$
\mu(a, m, c)=r(a, m, c) E[1 / \tau]=(m-a-c) \int_{0}^{\infty} \frac{1}{t} f(t ; m, a) d t
$$


Hereby, the density $f$ of the total trade time needs to be specified. The cumulative distribution function for the first passage time has the following form

$$
G_{[a, m]}\left(t \mid x_{0}, t_{0}\right)=1-\int_{a}^{m} \frac{1}{t} p\left(x, t \mid x_{0}, t_{0}\right) d t
$$

for transition densities $p\left(x, t \mid x_{0}, t_{0}\right)$ satisfying the Fokker-Planck equation

$$
\frac{\partial p(x, t)}{\partial t}=-\frac{\partial}{\partial x}(\mu(x, t) p(x, t))+\frac{\partial^{2}}{\partial x^{2}}\left(\frac{1}{2} \sigma^{2}(x, t) p(x, t)\right)
$$

Since the total trading time can be expressed in terms of Fokker-Planck equations, i.e., partial differential equations, the optimization problem can be solved numerically without necessity of simulation based methods. As opposed to the classic OU process, the general Itô diffusion does not allow for analytic results. The author presents two measures for the trade drawdown, i.e., the maximum negative market-to-market return during a trade, and suggests to incorporate them as constraints in the optimization problem. Summarizing, Bertram (2009) applies a powerful and flexible stochastic spread model, which includes a multitude of special cases. Hereby, the study reveals the following directions for further research. First, considering trade drawdown in the strategy is promising and has yet been integrated only by Bertram (2009) and Temnov (2015). Second, the author himself suggests to apply the strategy to the continuous-time random walk (CTRW), which is more realistic since it is non-Gaussian ${ }^{13}$.

\section{Other models}

This section summarizes other models with a limited set of supporting literature. The respective studies are listed in Table 6.

\subsection{Doubly mean-reverting model}

Liu et al. (2017) introduce a "doubly mean-reverting" model for intraday trading strategies, which is constructed as follows. In the first step, the long-term stochastic trend of the spread $X_{t}$, denoted by $L_{t}$, is modeled as a stochastic process. Specifically, $L_{t}$ follows an OU process with mean 0

$$
d L_{t}=-\theta_{L} L_{t} d t+\sigma_{L} d W_{t}^{L}
$$

\footnotetext{
${ }^{13}$ Osmekhin and Déleze (2015a) and Osmekhin and Déleze (2015b) use a CTRW for spread modeling. In this way, they overcome the drawback of the normal distribution. Their model can be seen as a generalization of the GBM with fat tails.
} 
Table 6

Other models

\begin{tabular}{|c|c|}
\hline Model & Study \\
\hline \multicolumn{2}{|c|}{ 6.1. Doubly mean-reverting model } \\
\hline \multicolumn{2}{|l|}{ Analytic approach } \\
\hline $\begin{array}{l}\text { Model-driven decisions: Optimizing formation and } \\
\text { trading period }\end{array}$ & Liu et al. (2017) \\
\hline \multicolumn{2}{|c|}{ 6.2. Nonlinear mean-reverting model } \\
\hline \multicolumn{2}{|l|}{ Dynamic programming } \\
\hline $\begin{array}{l}\text { Optimal investment allocation: Optimal portfolio } \\
\text { holdings under logarithmic utility }\end{array}$ & Alsayed and McGroarty (2013) \\
\hline \multicolumn{2}{|l|}{ Other approaches } \\
\hline Principal component analysis: Multi-factor approach & Avalon et al. (2017) \\
\hline \multicolumn{2}{|c|}{ 6.3. Skew mean-reverting model } \\
\hline \multicolumn{2}{|l|}{ Analytic approach } \\
\hline $\begin{array}{l}\text { Trading framework: Skewness, heavy tails, } \\
\text { and nonlinear mean-reversion }\end{array}$ & Avonleghi and Davison (2017) \\
\hline
\end{tabular}

In the second step, $X_{t}$ is modeled via a mean-reverting process around $L_{t}$ using the conditional modeling technique. Specifically, the spread $X_{t}$ follows

$$
d X_{t}=\theta\left(\tilde{L}(t)-X_{t}\right) d t+\sigma d W_{t}
$$

with mean process $\tilde{L}(t)=\frac{L_{2 i-2}+L_{2 i-1}}{2}$ and daily opening and closing values $L_{2 i-2}$ and $L_{2 i-1}$ of $X_{t}$ for day $i, i=1, \ldots, N$. As such, it is assumed that during any trading day, the spread reverts to the average of the current day's opening value and the previous day's closing value - a plausible, yet very restrictive assumption. The model is justified from a pairs selection perspective - pairs with stable $L_{t}$ and volatile $X_{t}$ are suitable pairs for trading.

Liu et al. (2017) optimize the pairs trading strategy based on the calibrated model of equations (46) and (47) in two respects. In the formation period, pairs are selected by high short-term and low long-term model variance. Trading candidates thus produce many trading opportunities while being stable in the longrun. In the trading period, a pairs trade is opened, when the spread deviates by from $L_{t}$, where is the $98 \%$ percentile of the absolute daily change in $L_{t}$ in the past 100 days. The position is closed when the spread reverts back to its mean level. Compared to existing studies, Liu et al. (2017) include two main improvements 
in their model. First, the spread reverts around a stochastic mean instead of a constant level or a linear function of time. Second, the stochastic long-term mean is itself a mean-reverting process, which distinguishes their work from the two-factor models in subsection 5.2. For further research, there are three main issues. First, future work should calibrate the stochastic mean level to a more complex function or process, replacing the simple step function calculated from daily opening and closing values. Second, the threshold is parametric and should instead be optimized by the model. Third, the authors themselves suggest to replace the Brownian motion by a more general Lévy process.

\subsection{Nonlinear mean-reverting model}

In a nonlinear generalization of the classic OU process (see Wong 1964, Alsayed and McGroarty 2013), the spread $X_{t}$ evolves according to

$$
d X_{t}=-\frac{\theta}{c} \tanh \left(c\left(X_{t}-\mu\right)\right) d t+\sigma d W_{t}
$$

with parameter $c>0$ modeling the nonlinearity of mean-reversion. For $c \rightarrow 0$, the model reduces to the OU process with mean-reversion rate $\theta$ and mean level $\mu$.

The nonlinear model of equation (48) differs from the classic OU model as follows. In the classic OU model, the mean-reversion strength increases linearly when the spread deviates from its mean. Consequently, the larger the deviations are, the more attractive the investment opportunities become. This is dangerous if spreads deviate far from their mean and the mean-reverting property actually does not persist (see Avalon et al. 2017). In the nonlinear OU model instead, the mean-reversion rate increases less when the spread deviates from its mean. Consequently, large deviations become more risky and less interesting.

Table 6 provides an overview of the relevant works applying a nonlinear mean-reverting model to capture the spread dynamics. As opposed to the previous sections, no analytic approach has been published.

Optimal investment allocation. Alsayed and McGroarty (2013) solve a portfolio optimization problem for an investor that allocates capital to a spread $X_{t}$, following equation (48), or a risk-free asset with rate $r$. The number of units held in the spread is denoted by $\boldsymbol{b}_{t}$. An investor with logarithmic utility solves the following portfolio optimization problem:

$$
\max _{b_{t}} E\left[\ln V_{t}\right]
$$

with wealth $V_{t}$. The optimal strategy is derived in closed form as follows:

$$
h_{t}\left(X_{t}, V_{t}\right)=\frac{-\frac{\theta}{c} \tanh \left(c\left(X_{t}-\mu\right)\right)-r X_{t}}{\sigma^{2}} V_{t}
$$


An analog specification of the optimal portfolio holdings for the classic OU model is derived in Jurek and Yang (2007), see equation (12). Due to the nonlinearity of mean-reversion in the nonlinear model, the capital allocation to diverging spreads is reduced compared to the classic OU model - losing trades are unwound sooner and the optimal portfolio holdings (see equation 49) decrease. Alsayed and McGroarty (2013) present the first academic study that explains the mean-reverting spread in a nonlinear model. As such, they provide an interesting new direction in pairs trading, yielding insights into the behavior of investors, especially concerning the capital allocation when large mispricings occur.

Principal component analysis. Based on the nonlinear OU process (48), Avalon et al. (2017) use a multi-factor statistical arbitrage model which incorporates methods such as principal component analysis and $\boldsymbol{k}$-means clustering. To calculate the optimal portfolio allocation to a pair, the authors use equation (49) for the nonlinear OU process and equation (12) derived by Jurek and Yang (2007) for the classic OU process as a benchmark. Compared to previous studies, the authors do not rely only on price data but also include time-series data on fundamentals in order to select better pairs for trading. As such, key contributions in this promising framework are the consolidation of multiple approaches and the integration of additional fundamental factors beyond classic price data.

\subsection{Skew mean-reverting model}

Avonleghi and Davison (2017) introduce another nonlinear generalization of the classic OU process. The spread $X_{t}$ follows

$$
d X_{t}=\theta\left(\mu-\frac{X_{t}}{2+\left|X_{t}\right|}\right) d t+\sigma d W_{t}
$$

and is mean-reverting for $-1<\mu<1$ with mean-reversion rate $\theta$, volatility $\sigma$, standard Brownian motion $\left\{W_{t}\right\}_{t \geq 0}$, and a long-run mean depending on all model parameters. According to Avonleghi and Davison (2017), the two main advantages of the model for pairs trading are the following. First, it is capable of capturing kurtosis and skewness in the transition density of the spread - both well-known stylized facts of financial data. Second, the strength of mean-reversion does not increase linearly as the process diverges away from its equilibrium level - instead, a slower increase of the mean-reversion strength compared to the classic OU process reflects the risk of diverging spreads. Despite the model's flexibility compared to the classic OU process, it does not have an analytic transition density and numerical methods need to be used for parameter estimation.

In the pairs trading literature, this flexible model has only been applied once by Avonleghi and Davison (2017) (see Tab. 6). The authors provide analytic results 
Review of stochastic differential equations in statistical arbitrage pairs trading

concerning the solution of the process as well as its stationary distribution. However, the development of a specific trading strategy is left for further research.

\section{Conclusion}

We have provided a comprehensive literature review of stochastic differential equations in statistical arbitrage pairs trading. The key findings and potential gaps regarding the five major spread models can be summarized as follows.

Section 2 covers the OU model to explain the spread dynamics - the majority of surveyed studies uses this model. The advantages are tractable analytic solutions, e.g., explicit trading thresholds derived by Bertram (2010b), optimal portfolio weights derived by Jurek and Yang (2007) and Mudchanatongsuk et al. (2008), and the ability to model mean-reversion. However, stylized facts of financial return series, especially in high-frequency settings, require extended stochastic spread models beyond the classic OU model.

Section 3 focuses on extended OU models. Correlations among multiple spreads are incorporated in multivariate OU frameworks. To the present day, there remains a lack of studies in this context, even though multivariate modeling is highly relevant from a practical perspective. The existence of jumps and fat tails is taken into account by Lévy driven OU models. Many studies confirm the non-normality of spread series, especially on small time scales (see Jondeau et al. 2015, Liu et al. 2017, Stübinger and Endres 2018). As such, Lévy driven OU models constitute an important extension of classic OU models driven by Brownian motion. However, in pairs trading they only emerged over the past few years. Research should aim at achieving further closed-form results for these Lévy driven OU models. Regime switching OU models are attractive since the allow parameters to vary across regimes. Just like the Lévy driven OU models, they only arose recently in continuous-time pairs trading and not much research has been done in that area. For further research, the suitability of such models in the high-frequency context should be analyzed. Bogomolov (2013) doubts whether it is possible to recognize switches and new parameters of the spread process quickly enough to adapt the trading strategy to changing market conditions.

Section 4 covers advanced mean-reverting diffusion models, i.e., CIR, IGBM, and SV models, which provide increased flexibility in the diffusion term of the model while their drift term is identical to the OU process. However, there remains a lack of literature exploiting this advancement and justifying the selection of these models for pairs trading. It should be investigated whether the CIR model including the property of non-negativity is applicable for stock price spreads from 
an empirical point of view. Then, analytic formulas, e.g., provided by Gregory et al. (2010), should be applied to large empirical data sets. Further, the advanced mean-reverting diffusion models could be extended from a model-perspective, e.g., by incorporating Lévy noise or regime-switching.

Section 5 focuses on diffusion models with a non-stationary component. Bertram (2009) introduces non-stationary spread models for continuous-time pairs trading to explain effects besides the classic mean-reversion property. However for non-stationary time-series, it is more difficult to distinguish extreme events, which trigger entry and exit decisions in pairs trading, from non-stationary shifts in the data (see Bertram 2010a). Recently, Fouque et al. (2016) and Tie et al. (2017) drop the classic mean-reverting property of pairs trading and model two stocks separately by GBMs. This provides a new direction in pairs trading and expands the range of potential pairs with desirable properties beyond the classic mean- reverting pairs. For further research, the GBM model could be expanded for a regime- switching component as proposed by Tie et al. (2017) or for a jump component as presented in Göncü and Akyildirim (2014). The two-factor model gains attention recently (see, e.g., Farkas et al. 2017 and Hahn et al. 2018), providing additional flexibility by allowing for two stochastic factors, e.g., short-term variations and long-term behavior. The two factors can be estimated from two different data bases (see Schwartz and Smith 2000). As such, the model is particularly interesting when considering additional data besides classic stock prices, e.g., sentiment, fundamentals, or cryptocurrency data. The general Itô diffusion model is highly flexible and includes many special cases - however, it has only been considered once in literature by Bertram (2009).

Section 6 covers other models with a limited set of supporting literature. In recent years, several studies have exploited new models beyond the classic ones, e.g., the doubly mean- reverting model by Liu et al. (2017), the nonlinear meanreverting model by Alsayed and McGroarty (2013), and the skew mean-reverting model by Avonleghi and Davison (2017). These models seem promising since they eliminate drawbacks of the classic OU process, which has been thoroughly discussed for more than a decade now. Further work should focus on the spread's fat tails, especially in high-frequency settings, combined with potential further factors that drive price dynamics.

Overall, we have comprehensively and critically reviewed the existing literature on stochastic differential equations in statistical arbitrage pairs trading - clearly, there remain gaps for further research and future studies. While the class of classic OU models has been extensively investigated, other models have only gained attention in recent years. Given the five main categories of stochastic spread models identified in this paper, there still exist other models apart from the aforementioned, e.g., two- and three-factor models with additional stochastic components. 


\section{References}

[1] Ackerer, D., Filipović, D. and Pulido, S. (2018) 'The Jacobi stochastic volatility model', Finance and Stochastics, vol. 22, pp. 667-700.

[2] Alsayed, H. and McGroarty, F. (2013) 'Optimal Portfolio Selection in Nonlinear Arbitrage Spreads', The European Journal of Finance, vol. 19, pp. 206-227.

[3] Altay, S., Colaneri, K. and Eksi, Z. (2017) 'Pairs trading under drift uncertainty and risk penalization', Working Paper, Vienna University of Technology.

[4] Angoshtari, B. (2016) 'On the market-neutrality of optimal pairs trading strategies', Working paper, University of Washington.

[5] Avalon, G., Becich, M., Cao, V., Jeon, I., Misra, S. and Puzon, L. (2017) 'Multifactor statistical arbitrage model', Working paper, Stanford University.

[6] Avellaneda, M. and Lee, J.H. ( 2010) 'Statistical Arbitrage in the U.S. Equities Market', Quantitative Finance, vol. 10, pp. 761-782.

[7] Avonleghi, M.H. and Davison, M. (2017) 'Modeling energy spreads with a generalized novel mean-reverting stochastic process', Journal of Energy Markets, Forthcoming.

[8] Bachelier, L. (1900) Théorie de la spéculation, Paris: Gauthier-Villars.

[9] Bai, Y. and Wu, L. (2018) 'Analytic value function for optimal regime-switching pairs trading rules', Quantitative Finance, vol. 18, pp. 637-654.

[10] Baronyan, S.R., Bodurğlu, İ.İ., Şener, E. (2010) 'Investigation of Stochastic Pairs Trading Strategies Under Different Volatility Regimes', The Manchester School, vol. 78, pp. 114-134.

[11] Baviera, R. and Baldi, T.S. (2018) 'Stop-loss and leverage in optimal statistical arbitrage with an application to energy market', Energy Economics, Forthcoming.

[12] Bertram, W.K. (2009) 'Optimal trading strategies for Itô diffusion processes', Physica A: Statistical Mechanics and its Applications, vol. 388, pp. 2865-2873.

[13] Bertram, W.K. (2010a) 'A non-stationary model for statistical arbitrage trading', Working Paper, Columbia University, doi:10.2139/ssrn.1632718.

[14] Bertram, W.K. (2010b) 'Analytic solutions for optimal statistical arbitrage trading', Physica A: Statistical Mechanics and its Applications, vol. 389, pp. 2234-2243, doi:10.1016/j.physa.2010.01.045.

[15] Blázquez, M.C. and Román, C. (2018) 'Pairs trading techniques: An empirical contrast', European Research on Management and Business Economics, vol. 24, pp. 160-167.

[16] Bock, M., Mestel, R. (2009) 'A regime-switching relative value arbitrage rule', in Fleischmann, B., Borgwardt, K., Klein, R., Tuma, A. (eds.) Operations Research Proceedings 2008, Berlin: Springer, pp. 9-14.

[17] Bodo, B.A., Thompson, M.E. and Unny, T.E. (1987) 'A review on stochastic differential equations for applications in hydrology', Stochastic Hydrology and Hydraulics, vol. 1, pp. 81-100, doi:10.1007/BF01543805. 
[18] Bogomolov, T. (2011) 'Pairs trading in the land down under', in Finance and Corporate Governance Conference, Melbourne.

[19] Bogomolov, T. (2013) 'Pairs trading based on statistical variability of the spread process', Quantitative Finance, vol. 13, pp. 1411-1430, doi:10.108 0/14697688.2012.748934.

[20] Boguslavsky, M. and Boguslavskaya, E. (2004) 'Arbitrage under power', Risk, vol. 17, pp. 69-73.

[21] Bucca, A. and Cummins, M. (2011) 'Synthetic floating crude oil storage and optimal statistical arbitrage: A model specification analysis', Working Paper, Dublin City University Business School.

[22] Burks, N., Li, B., Bowling, J. and Schmid, N. (2018) 'Capitalizing Off Collapse: The Hidden Alpha During the Crisis Periods', International Research Journal of Applied Finance, vol. 9, pp. 124-133.

[23] Cartea, Á. and Jaimungal, S. (2016) 'Algorithmic Trading of Co-Integrated Assets', International Journal of Theoretical and Applied Finance, vol. 19, pp. 1-18.

[24] Chanol, E., Collet, O., Kostyuchyk, N., Mesbah, T., Nguyen and Q.H.L. (2015) 'Co-integration for Soft Commodities with Non Constant Volatility', International Journal of Trade, Economics and Finance, vol. 6, pp. 32-36.

[25] Charalambous, K., Sophocleous, C., O'Hara, J.G. and Leach, P.G. (2015) 'A deductive approach to the solution of the problem of optimal pairs trading from the viewpoint of stochastic control with time-dependent parameters', Matbematical Methods in the Applied Sciences, vol. 38, pp. 4448-4460.

[26] Chiu, M.C. and Wong, H.Y. (2011) 'Mean-variance portfolio selection of cointegrated assets', Journal of Economic Dynamics and Control, vol. 35, pp. 1369-1385.

[27] Chiu, M.C. and Wong, H.Y. (2015) 'Dynamic cointegrated pairs trading: Mean-variance time-consistent strategies', Journal of Computational and Applied Mathematics, vol. 290, pp. 516-534, doi:10.1016/j.cam.2015.06.004.

[28] Chiu, M.C. and Wong, H.Y. (2018) 'Robust dynamic pairs trading with cointegration', Operations Research Letters, vol. 46, pp. 225-232.

[29] Cont, R. (2001) 'Empirical properties of asset returns: stylized facts and statistical issues', Quantitative Finance, vol. 1, pp. 223-236.

[30] Cummins, M. (2010) 'Optimal statistical arbitrage: A model specification analysis on ISEQ equity data', Irish Accounting Review, vol. 17, pp. 21-40.

[31] Cummins, M. and Bucca, A. (2012) 'Quantitative Spread Trading on Crude Oil and Refined Products Markets', Quantitative Finance, vol. 12, pp. 1857-1875.

[32] D'Aspremont, A. (2011) 'Identifying Small Mean Reverting Portfolios', Quantitative Finance, vol. 11, pp. 351-364, doi:10.1080/14697688.2010.481634.

[33] Diamond, R. (2014) 'Learning and trusting cointegration in statistical arbitrage', Working Paper, CQF Institute, New York. 
[34] Do, B., Faff, R. and Hamza, K. (2006) 'A new approach to modeling and estimation for pairs trading', in Proceedings of 2006 Financial Management Association European Conference, Stockholm, Sweden.

[35] Dourban, A. and Yedidsion, L. (2015) 'Threshold function for the optimal stopping of arithmetic Ornstein-Uhlenbeck process', in Proceedings of the 2015 International Conference on Operations Excellence and Service Engineering, pp. 1-7.

[36] Dunis, C.L., Giorgioni, G., Laws, J. and Rudy, J. (2010) 'Statistical arbitrage and high-frequency data with an application to Eurostoxx 50 equities', Working Paper, Liverpool Business School.

[37] Einstein, A. (1905) 'Über die von der molekularkinetischen Theorie der Wärme geforderte Bewegung von in ruhenden Flüssigkeiten suspendierten Teilchen', Annalen der Pbysik, vol. 322, pp. 549-560.

[38] Ekström, E., Lindberg, C. and Tysk, J. (2011) 'Optimal liquidation of a pairs trade', in Di Nunno, G., Øksendal, B. (eds.), Advanced Mathematical Methods for Finance, Springer, Berlin, Germany and Heidelberg, pp. 247-255.

[39] Elliott, R.J., van der Hoek, J. and Malcolm, W.P. (2005) 'Pairs trading', Quantitative Finance, vol. 5, pp. 271-276.

[40] Endres, S. and Stübinger, J. (2019a) 'A flexible regime switching model with pairs trading application to the S\&P 500 high-frequency stock returns', Quantitative Finance, vol. 19, pp. 1727-1740.

[41] Endres, S. and Stübinger, J. (2019b) 'Optimal trading strategies for Lévy-driven Ornstein-Uhlenbeck processes', Applied Economics, vol. 51, pp. 3153-3169.

[42] Fanelli, V. and Lesca, A. (2014) 'Natural gas statistical arbitrage', New Frontiers in Practical Risk Management, vol. 61, pp. 61-70.

[43] Farkas, W., Gourier, E., Huitema, R. and Necula, C. (2017) 'A two-factor cointegrated commodity price model with an application to spread option pricing', Journal of Banking \& Finance, vol. 77, pp. 249-268.

[44] Figuerola-Ferretti, I., Paraskevopoulos, I. and Tang, T. (2015) 'Market model for valuing and managing portfolio strategies', Working Paper, University of Madrid.

[45] Figuerola-Ferretti, I., Paraskevopoulos, I. and Tang, T. (2017) 'A market approach for convergence trades', Working Paper, University of Madrid.

[46] Focardi, S.M., Fabozzi, F.J. and Mitov, I.K. (2016) 'A new approach to statistical arbitrage: Strategies based on dynamic factor models of prices and their performance', Journal of Banking E Finance, vol. 65, pp. 134-155, doi:10.1016/j.jbankfin.2015.10.005.

[47] Fouque, J.P., Pun, C.S. and Wong, H.Y. (2016) 'Portfolio Optimization with Ambiguous Correlation and Stochastic Volatilities', SIAMJournal on Control and Optimization, vol. 54, pp. 2309-2338.

[48] Gatev, E., Goetzmann, W.N. and Rouwenhorst, K.G. (1999) 'Pairs trading: Performance of a relative value arbitrage rule', Working Paper, Yale School of Management's International Center for Finance. 
[49] Gatev, E., Goetzmann, W.N. and Rouwenhorst, K.G. (2006) 'Pairs Trading: Performance of a Relative-Value Arbitrage Rule', Review of Financial Studies, vol. 19, pp. 797-827.

[50] Göncü, A. (2015) 'Statistical Arbitrage in the Black-Scholes Framework', Quantitative Finance, vol. 15, pp. 1489-1499.

[51] Göncü, A. and Akyildirim, E. (2014) 'Statistical Arbitrage in jump-diffusion models with compound Poisson processes', Working Paper, Liverpool University, doi:10.2139/ssrn.2542912.

[52] Göncü, A. and Akyildirim, E. (2016a) 'A stochastic model for commodity pairs trading', Quantitative Finance, vol. 16, pp. 1843-1857, doi:10.1080/ 14697688.2016.1211793.

[53] Göncü, A. and Akyildirim, E. (2016b) 'Statistical Arbitrage with Pairs Trading', International Review of Finance, vol. 16, pp. 307-319.

[54] Göncü, A. and Akyildirim, E. (2017) 'Statistical arbitrage in the multi-asset Black-Scholes economy', Annals of Financial Economics, vol. 12, pp. 1-19.

[55] Gregory, I., Ewald, C.O. and Knox, P. (2010) 'Analytical pairs trading under different assumptions on the spread and ratio dynamics', in 23rd Australasian Finance and Banking Conference, Sydney, doi:10.2139/ssrn.1663703.

[56] Gu, J. and Steffensen, M. (2015) 'Optimal portfolio liquidation and dynamic mean-variance criterion', Working Paper, The University of Hong Kong.

[57] Hahn, W.J., DiLellio, J.A. and Dyer, J.S. (2018) 'Risk premia in commodity price forecasts and their impact on valuation', Energy Economics, vol. 72, pp. 393-403.

[58] Heston, S.L. (1993) 'A Closed-Form Solution for Options with Stochastic Volatility with Applications to Bond and Currency Options', Review of Financial Studies, vol. 6, pp. 327-343.

[59] Jondeau, E., Lahaye, J. and Rockinger, M. (2015) 'Estimating the price impact of trades in a high-frequency microstructure model with jumps', Journal of Banking E Finance, vol. 61, pp. 205-224.

[60] Jurek, J.W. and Yang, H. (2007) Dynamic portfolio selection in arbitrage, Working Paper, Harvard University, doi:10.2139/ssrn.882536.

[61] Kanamura, T., Rachev, S.T. and Fabozzi, F.J. (2010) 'A profit model for spread trading with an application to energy futures', The Journal of Trading, vol. 5, pp. 48-62.

[62] Kang, J., Leung, T. (2017) 'Asynchronous ADRs: overnight vs intraday returns and trading strategies', Studies in Economics and Finance, vol. 34, pp. 580-596.

[63] Kim, K. (2011) 'Performance analysis of pairs trading strategy utilizing high frequency data with an application to KOSPI 100 equities', Working Paper, Harvard University, doi:10.2139/ssrn.1913707.

[64] Kim, S.J., Primbs, J. and Boyd, S. (2008) 'Dynamic spread trading', Working Paper, Stanford University. 
[65] Kitapbayev, Y. and Leung, T. (2017) 'Optimal mean-reverting spread trading: nonlinear integral equation approach', Annals of Finance, vol. 13, pp. 181-203.

[66] Kitapbayev, Y. and Leung, T. (2018) 'Mean Reversion Trading with Sequential Deadlines and Transaction Costs', International Journal of Theoretical and Applied Finance, vol. 21, pp. 1-22.

[67] Krauss, C. (2017) 'Statistical arbitrage pairs trading strategies: Review and outlook', Journal of Economic Surveys, vol. 31, pp. 513-545, doi:10.1111/ joes. 12153.

[68] Kuo, K., Luu, P., Nguyen, D., Perkerson, E., Thompson, K. and Zhang, Q. (2015) 'Pairs trading: An optimal selling rule', Matbematical Control and Related Fields, vol. 5, pp. 489-499, doi:10.3934/mcrf.2015.5.489.

[69] Larsson, S., Lindberg, C., Warfheimer, M. (2013) 'Optimal closing of a pair trade with a model containing jumps', Applications of Mathematics, vol. 58, pp. 249-268.

[70] Lei, Y. and $\mathrm{Xu}, \mathrm{J}$. (2015) 'Costly arbitrage through pairs trading', Journal of Economic Dynamics and Control, vol. 56, pp. 1-19, doi:10.1016/ j.jedc.2015.04.006.

[71] Leung, T. and Li, X. (2015) 'Optimal Mean Reversion Trading with Transaction Costs and Stop-Loss Exit', International Journal of Theoretical and Applied Finance, vol. 18, pp. 1-31.

[72] Leung, T. and $\mathrm{Li}, \mathrm{X}$. (2016) 'Optimal mean reversion trading: Mathematical analysis and practical applications', World Scientific, Singapore, doi: $10.1142 / 9839$.

[73] Li, T.N. and Tourin, A. (2016) 'Optimal Pairs Trading with Time-Varying Volatility', International Journal of Financial Engineering, vol. 3, pp. 1-45.

[74] Li, X. (2015) Optimal multiple stopping approach to mean reversion trading, Ph.D. thesis, Columbia University, doi:10.7916/D88K781S.

[75] Lindberg, C. (2014) 'Pairs trading with opportunity cost', Journal of Applied Probability, vol. 51, pp. 282-286.

[76] Lintilhac, P.S. and Tourin, A. (2017) 'Model-based pairs trading in the bitcoin markets', Quantitative Finance, vol. 17, pp. 703-716.

[77] Liu, B., Chang, L.B. and Geman, H. (2017) 'Intraday pairs trading strategies on high frequency data: the case of oil companies', Quantitative Finance, vol. 17, pp. 87-100, doi:10.1080/14697688.2016.1184304.

[78] Liu, J. and Timmermann, A. (2013) 'Optimal Convergence Trade Strategies', Review of Financial Studies, vol. 26, pp. 1048-1086.

[79] Meucci, A. (2009) 'Review of Statistical Arbitrage, Cointegration, and Multivariate Ornstein-Uhlenbeck', Working Paper, Symmys, pp. 1-20.

[80] Mota, P.P. and Esquivel, M.L. (2016) 'Model selection for stock prices data', Journal of Applied Statistics, vol. 43, pp. 2977-2987, doi:10.1080/026647 63.2016.1155205. 
[81] Mudchanatongsuk, S., Primbs, J.A. and Wong, W. (2008) 'Optimal Pairs Trading: A Stochastic Control Approach', in American Control Conference, 2008, pp. 1035-1039.

[82] Ngo, M.M. and Pham, H. (2016) 'Optimal switching for the pairs trading rule: a viscosity solutions approach',Journal of Mathematical Analysis and Applications, vol. 441, pp. 403-425.

[83] Nóbrega, J.P. and Oliveira, A.L. (2013) 'Improving the Statistical Arbitrage Strategy in Intraday Trading by Combining Extreme Learning Machine and Support Vector Regression with Linear Regression Models', in IEEE 25th International Conference on Tools with Artificial Intelligence, pp. 182-188.

[84] Nobrega, J.P. and Oliveira, A.L. (2014) 'A combination forecasting model using machine learning and kalman filter for statistical arbitrage', in IEEE International Conference on Systems, Man and Cybernetics, pp. 1294-1299.

[85] Osmekhin, S. and Déleze, F. (2015a) 'Application of continuous-time random walk to statistical arbitrage', Journal of Engineering Science and Technology Review, vol. 8, pp. 91-95.

[86] Osmekhin, S. and Déleze, F. (2015b) 'Waiting-time distribution and market efficiency: Evidence from statistical arbitrage', Working Paper, Hanken School of Economics.

[87] Pilipovic, D. (2007) Energy Risk: Valuing and Managing Energy Derivatives. New York: McGraw Hill Professional.

[88] Psaradellis, I., Laws, J., Pantelous, A.A. and Sermpinis, G. (2018) 'Pairs trading, technical analysis and data snooping: Mean reversion vs. momentum', Working Paper, University of St. Andrews, doi:10.2139/ssrn.3128788.

[89] Rampertshammer, S. (2007) 'An Ornstein-Uhlenbeck framework for pairs trading', Working Paper, University of Melbourne.

[90] Schwartz, E. (1997) 'The Stochastic Behavior of Commodity Prices: Implications for Valuation and Hedging', The Journal of Finance, vol. 52, pp. 923-973.

[91] Schwartz, E. and Smith, J. (2000) 'Short-Term Variations and Long-Term Dynamics in Commodity Prices', Management Science, vol. 46, pp. 893-911.

[92] Sharp, K.P. (1990) 'Stochastic differential equations in finance'. Applied Mathematics and Computation, vol. 37, pp. 131-148.

[93] Smoluchowski, M. von (1906) 'Zur kinetischen Theorie der brownschen Molekularbewegung und der Suspensionen', Annalen der Pbysik, vol. 326, pp. 756-780.

[94] Song, Q. and Zhang, Q. (2013) 'An optimal pairs-trading rule', Automatica, vol. 49, pp. 3007-3014, doi:10.1016/j.automatica.2013.07.012.

[95] Stehlıková, B., (2008) Mathematical analysis of term structure models, Ph.D. thesis, Comenius University Bratislava.

[96] Stübinger, J. and Endres, S. (2018) 'Pairs trading with a mean-reverting jumpdiffusion model on high-frequency data', Quantitative Finance, vol. 18, pp. 1735-1751. 
[97] Suzuki, K. (2016) 'Optimal switching strategy of a mean-reverting asset over multiple regimes', Automatica, vol. 67, pp. 33-45.

[98] Suzuki, K. (2018) 'Optimal pair-trading strategy over long/short/square positions - empirical study', Quantitative Finance, vol. 18, pp. 97-119.

[99] Temnov, G. (2015) 'Analysis of Ornstein-Uhlenbeck process stopped at maximum drawdown and application to trading strategies with trailing stops', Working Paper, Charles University, Prague, pp. 1-19.

[100] Thiele, T.N. (1880) Om Anvendelse af mindste Kvadraters Methode i nogle Tilfælde, hvor en Komplikation af visse Slags uensartede tilfældige Fejlkilder giver Fejlene en 'systematisk' Karakter. Det Kongelige Danske Videnskabernes Selskabs Skrifter-Naturvidenskabelig og Mathematisk Afdeling, pp. 381-408.

[101] Tie, J., Zhang, H. and Zhang, Q. (2017) 'An optimal strategy for pairs trading under geometric Brownian motions', Journal of Optimization Theory and Applications, pp. 1-22.

[102] Tourin, A. and Yan, R. (2013) 'Dynamic pairs trading using the stochastic control approach', Journal of Economic Dynamics and Control, vol. 37, pp. 1972-1981.

[103] Triantafyllopoulos, K. and Montana, G. (2011) 'Dynamic modeling of meanreverting spreads for statistical arbitrage', Computational Management Science, vol. 8, pp. 23-49.

[104] Wissner-Gross, A.D. and Freer, C.E. (2010) 'Relativistic statistical arbitrage', Physical Review E, vol. 82, pp. 1-7.

[105] Wong, E. (1964) 'The construction of a class of stationary Markoff processes', Stochastic Processes in Mathematical Physics and Engineering, vol. 17, pp. 264-276.

[106] Yamamoto, R. and Hibiki, N. (2017) 'Optimal multiple pairs trading strategy using derivative free optimization under actual investment management conditions', Journal of the Operations Research Society of Japan, vol. 60, pp. 244-261.

[107] Yang, J.W., Tsai, S.Y., Shyu, S.D. and Chang, C.C. (2016) 'Pairs trading: The performance of a stochastic spread model with regime switching-evidence from the S\&P 500', International Review of Economics \& Finance, vol. 43, pp. 139-150, doi:10.1016/j.iref.2015.10.036.

[108] Yang, Y., Göncü, A. and Pantelous, A. (2017) 'Pairs Trading with Commodity Futures: Evidence from the Chinese Market', China Finance Review International, vol. 7, pp. 274-294, doi:10.1108/CFRI-09-2016-0109.

[109] Yeo, J. and Papanicolaou, G. (2017) 'Risk control of mean-reversion time in statistical arbitrage', Risk and Decision Analysis, vol. 6, pp. 263-290.

[110] Yoshikawa, D. (2017) 'An Entropic Approach for Pair Trading', Entropy, vol. 19, pp. 320. 
[111] Zeng, Z. and Lee, C.G. (2014) 'Pairs trading: optimal thresholds and profitability', Quantitative Finance, vol. 14, pp. 1881-1893, doi:10.1080/14697688.2014. 917806.

[112] Zhang, H. and Zhang, Q. (2008) 'Trading a Mean-Reverting Asset: Buy Low and Sell High', Automatica, vol. 44, pp. 1511-1518.

[113] Zhang, J., Leung, T. and Aravkin, A.Y. (2018) Mean Reverting Portfolios via penalized OU-Likelihood Estimation, Working Paper, University of Washington.

[114] Zhao, B. (2009) 'Mean First Passage Times of the Feller and the GARCH Diffusion Processes', Working Paper, City University London, doi:10.2139/ ssrn. 1465334.

[115] Zhengqin, Z. (2014) 'Optimal Pairs Trading: Static and Dynamic Models', Working Paper, University of Toronto. 